\title{
Quantum Black Holes, Elliptic Genera and Spectral Partition Functions
}

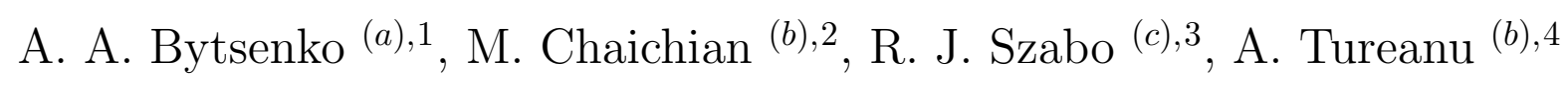 \\ (a) Departamento de Física, Universidade Estadual de Londrina \\ Caixa Postal 6001, Londrina-Paraná, Brazil \\ (b) Department of Physics, University of Helsinki \\ P.O. Box 64, FI-00014 Helsinki, Finland \\ (c) Department of Mathematics, Heriot-Watt University \\ Colin Maclaurin Building, Riccarton, Edinburgh EH14 4AS, UK \\ Maxwell Institute for Mathematical Sciences, Edinburgh, UK \\ The Tait Institute, Edinburgh, UK
}

\begin{abstract}
We study M-theory and D-brane quantum partition functions for microscopic black hole ensembles within the context of the AdS/CFT correspondence in terms of highest weight representations of infinite-dimensional Lie algebras, elliptic genera, and Hilbert schemes, and describe their relations to elliptic modular forms. The common feature in our examples lie in the modular properties of the characters of certain representations of the pertinent affine Lie algebras, and in the role of spectral functions of hyperbolic three-geometry associated with $q$-series in the calculation of elliptic genera. We present new calculations of supergravity elliptic genera on local Calabi-Yau threefolds in terms of BPS invariants and spectral functions, and also of equivariant D-brane elliptic genera on generic toric singularities. We use these examples to conjecture a link between the black hole partition functions and elliptic cohomology.
\end{abstract}

August 2013

EMPG-13-13

\footnotetext{
1 aabyts@gmail.com

${ }^{2}$ masud.chaichian@helsinki.fi

${ }^{3}$ R.J.Szabo@hw.ac.uk

4anca.tureanu@helsinki.fi
} 


\section{Contents}

1 Introduction $\quad 2$

2 Superconformal field theories, elliptic genera and symmetric products 4 2.1 Elliptic genera . . . . . . . . . . . . . . . . . . . . . . 4

2.2 Superconformal sigma-models on symmetric products . . . . . . . . . 9 9

2.3 Hilbert schemes and representations of affine Lie algebras . . . . . . . . . 10

3 M-theory partition functions and $(4,0)$ elliptic genera 12

3.1 Black hole partition functions in the M-theory frame . . . . . . . . . 12

3.2 Quantum gravity and spectral partition functions on $A d S_{3}$. . . . . . . . 14

3.3 Supergravity boson-fermion correspondence . . . . . . . . . . 16

3.4 Supergravity elliptic genus on $A d S_{3} \times S^{2} \times X \ldots \ldots$. . . . . . . . . . 17

3.5 Spectral partition functions for BPS invariants . . . . . . . . . . 19

4 D-brane partition functions and $(2,2)$ elliptic genera 22

4.1 Black hole partition functions in the Type IIA frame . . . . . . . . . . 22

4.2 Black hole components of chiral primary states . . . . . . . . . . . . 24

4.3 Microscopic black hole entropy . . . . . . . . . . . . . . . . 26

4.4 Representations of Heisenberg algebras . . . . . . . . . . . . . 27

4.5 Toric Calabi-Yau black holes . . . . . . . . . . . . . . . 27

$4.6 \quad(2,2)$ field theory elliptic genus . . . . . . . . . . . . . 31

5 Conclusions

A Spectral functions of hyperbolic three-manifolds

B Singularities and poles of elliptic genera $\quad 38$

\section{Introduction}

In this paper we discuss applications of affine Lie algebra representations, elliptic genera and their generalizations, and Hilbert schemes to the computation of M-theory and Dbrane quantum partition functions for microscopic black hole ensembles, together with their connections to elliptic modular forms and spectral functions in the context of AdS/CFT holography. The main techniques we use involve the Grothendieck-Riemann-Roch theorem, characters of affine Lie algebras, and spectral functions of hyperbolic three-geometry. In particular, we make use of the characteristic classes of foliations of Lie group representations, 
which allow us to derive interesting new results and important old results in superconformal field theory.

Let us begin by explaining the general scheme. Suppose that $\mathfrak{g}$ is the Lie algebra of a Lie group $G$. Let us consider the pair $(H, G)$ of Lie groups, where $H$ is a closed subgroup of $G$ with normalizer subgroup $N_{H} \subset G$. Then the pair $(H, G)$ with the discrete quotient group $N_{H} / H$ corresponds to the inclusion $\mathfrak{g} \hookrightarrow W_{n}$, where $W_{n}$ is the Lie algebra of formal vector fields in $n=\operatorname{dim} G / H$ variables, while the homogeneous space $G / H$ possesses a canonical $\mathfrak{g}$-structure $\omega 1$ (see [24] for details). Combining this $\mathfrak{g}$-structure with the inclusion $\mathfrak{g} \hookrightarrow W_{n}$, one obtains a $W_{n}$-structure on the quotient space $G / \mathfrak{G}$ for any discrete subgroup $\mathfrak{G}$ of the Lie group $G$; this is precisely the $W_{n}$-structure which corresponds to the $H$-equivariant foliation of $G$ by left cosets of $\mathfrak{G}\left[24\right.$. The homomorphism $\operatorname{char}_{\omega}: H^{\bullet}\left(W_{n}\right) \rightarrow H^{\bullet}(G / \mathfrak{G}, \mathbb{R})$ associated with characteristic classes of $W_{n}$-structures decomposes into the composition of two homomorphisms $H^{\bullet}\left(W_{n}\right) \rightarrow H^{\bullet}(\mathfrak{g})$ and $H^{\bullet}(\mathfrak{g}) \rightarrow H^{\bullet}(G / \mathfrak{G}, \mathbb{R})$; the first homomorphism is independent of $\mathfrak{G}$ and is induced by the inclusion $\mathfrak{g} \hookrightarrow W_{n}$ (see e.g. [16]), while the second homomorphism is independent of $H$ and corresponds to the canonical homomorphism which determines the characteristic classes of the canonical $\mathfrak{g}$-structure $\omega$ on $G / \mathfrak{G}$. If the group $G$ is semisimple, then the Lie algebra $\mathfrak{g}$ is unitary and $G$ contains a discrete subgroup $\mathfrak{G}$ for which $G / \mathfrak{G}$ is compact; for appropriate choice of $\mathfrak{G}$ the kernel of the homomorphism $H^{\bullet}\left(W_{n}\right) \rightarrow H^{\bullet}(G / \mathfrak{G}, \mathbb{R})$ coincides with the kernel of the homomorphism $H^{\bullet}\left(W_{n}\right) \rightarrow H^{\bullet}(\mathfrak{g})$.

In our applications we shall consider a compact hyperbolic three-manifold $G / \mathfrak{G}$ with $G=$ $S L(2, \mathbb{C})$. By combining the characteristic class representatives of field theory elliptic genera with the homomorphism $\operatorname{char}_{\omega}$, one can compute the elliptic genera (and hence our quantum partition functions) in terms of the spectral functions of hyperbolic three-geometry. All of these structures arise quite naturally in string theory, and are particularly clear and tractable when supersymmetry is involved.

We shall now describe the specific contents of this paper, and their interrelationships with the general sheme described above. In the following we study examples of quantum black hole partition functions and show that all of them, although different in their nature, can be expressed in a generic way by means of spectral functions of hyperbolic three-geometry. This enables us to establish concrete modular properties of the partition functions even in instances that are not covered by $A d S_{3} / C F T_{2}$ holography, and thus it naturally explains the modularity in these cases. Along the way a number of new calculations of elliptic genera are presented.

A central concept in this paper is that of the elliptic genus and its generalizations which we introduce in Sect. 2, together with some pertinent examples. We analyze its realisation as one-loop partition functions of superconformal sigma-models on symmetric product orbifolds, and survey various features of the orbifold resolutions by Hilbert schemes, particularly the way in which they yield natural geometric constructions of representations of affine Lie algebras.

In Sect. 3 we consider microscopic black hole partition functions in M-theory on a suitable

\footnotetext{
${ }^{1}$ Following [6, by a $\mathfrak{g}$-structure on a smooth manifold $X$ we mean a smooth one-form $\omega$ on $X$ with values in $\mathfrak{g}$ which satisfies the Maurer-Cartan equation $\mathrm{d} \omega=-\frac{1}{2}[\omega, \omega]$, i.e. for any pair of vector fields $\xi_{1}, \xi_{2}$ on $X$, one has $\mathrm{d} \omega\left(\xi_{1}, \xi_{2}\right)=-\left[\omega\left(\xi_{1}\right), \omega\left(\xi_{2}\right)\right]$.
} 
attractor geometry in the context of the $A d S_{3} / C F T_{2}$ correspondence. We discuss its relationship to the minimal three-dimensional quantum gravity in a spacetime which is asymptotic to $A d S_{3}$; the symmetry group of $A d S_{3}$ gravity (with appropriate boundary conditions) is generated by the Virasoro algebra, and the one-loop partition function is indeed the partition function of a conformal field theory in two dimensions. We then derive in detail the supergravity elliptic genus on $A d S_{3} \times S^{2} \times X$ in terms of the Ruelle-type spectral functions, with $X$ a Calabi-Yau threefold; we explain how to rewrite these M-theory black hole partition functions geometrically in terms of BPS invariants and derive a spectral function formulation for it.

In Sect. 4 we analyze the black hole partition functions in the reduction to Type IIA string theory, and the realisation therein of the black hole components of chiral primary states of the minimal $\mathcal{N}=4$ superconformal algebra. The chiral primary fields of an $\mathcal{N}=2$ superconformal field theory form an algebra; the proof of this fact [42, 54] shares many common features with the Hodge theory of Kähler manifolds, and here the connection arises since the underlying D0-brane superconformal field theory is holographically dual to Type IIA string theory on $A d S_{2} \times S^{2} \times X$. We evaluate the generating function for chiral primary states in terms of the Ruelle-type spectral functions. The microscopic black hole entropy is then evaluated from various points of view including the one-loop partition function of a generalised sigma-model, the Hirzebruch $\chi_{y}$-genus, and the $(2,2)$ field theory elliptic genus. These results tie in to the M-theory analysis of Sect. 3 through the relationship between the equivariant genera of D-brane moduli spaces, which are given by Hilbert schemes, and the Gopakumar-Vafa expansion of topological string partition functions of some local CalabiYau geometries in terms of BPS invariants. In particular, we present new calculations of equivariant D-brane elliptic genera on generic toric singularities.

Finally, in Sect. 5 we briefly summarise our main conclusions from this analysis. Two appendices at the end of the paper are devoted to a brief definition and summary of the main features of the spectral functions of hyperbolic three-geometry, including their analytic properties, and their relations to the singularities of elliptic genera. From these facts, we conjecture an intimate connection between the black hole partition functions and elliptic cohomology.

\section{Superconformal field theories, elliptic genera and sym- metric products}

\section{$2.1 \quad$ Elliptic genera}

Elliptic genera are the natural topological invariants of manifolds which generalize the classical genera. They appear, for example, when one considers the supersymmetric indices of superconformal vertex algebras. For mathematicians, elliptic genera are regarded as invariants for spaces in a generalized cohomology theory extending K-theory, called elliptic cohomology. For physicists, elliptic genera are the one-loop string partition functions which capture important refined information about the spectrum of supersymmetric states of the 
underlying superconformal field theory. Moreover, when they are applied to instanton moduli spaces of supersymmetric gauge theories in four dimensions, they also encompass as special cases the Vafa-Witten partition function 55 for $\mathcal{N}=4$ gauge theory and Nekrasov's partition function [47] for $\mathcal{N}=2$ gauge theory (see e.g. [18] for a collection of results). In this paper we will exploit elliptic genera in calculating quantum black hole partition functions.

In physics, a two-variable elliptic genus can be associated with any two-dimensional $\mathcal{N}=$ $(2,2)$ superconformal field theory [20, 56, 36]; it is given by

$$
\operatorname{Tr}_{\mathcal{H}}(-1)^{F} y^{J_{0}} q^{L_{0}-c / 24} \bar{q}^{\bar{L}_{0}-c / 24}
$$

where $q$ and $y$ are complex parameters, $L_{0}$ (resp. $\bar{L}_{0}$ ) is the Virasoro generator of left (resp. right) movers, and $J_{0}$ is the $U(1)$ charge operator of left moving modes. The trace is taken over the states in the Ramond-Ramond sector of the Hilbert space $\mathcal{H}$ of the superconformal field theory, and $F=F_{L}+F_{R}$ with $F_{L}$ (resp. $F_{R}$ ) the fermion number of left (resp. right) movers. The elliptic genus is invariant under smooth variations of the parameters of the field theory and is useful for enumerating BPS states.

For a superconformal sigma-model having as target space a smooth compact complex manifold $X$ of dimension $d$, the genus one partition function is defined as the trace over the Ramond-Ramond sector of the time evolution operator $q^{H}$ times $(-1)^{F} y^{F_{L}}$. If $\mathcal{E}=\mathbb{C} /(\mathbb{Z}+$ $\mathbb{Z} \tau)$ is an elliptic curve with complex structure modulus $\tau \in \mathbb{C}^{+}$and the line bundle parametrized by $z \in \operatorname{Jac}(\mathcal{E}) \cong \mathcal{E}$, then we set $q:=\mathrm{e}^{2 \pi \mathrm{i} \tau}(\mathrm{i}:=\sqrt{-1})$ and $y:=\mathrm{e}^{2 \pi \mathrm{i} z}$. The field theory elliptic genus is then defined as [19]

$$
\chi(X ; q, y)=\operatorname{Tr} \operatorname{H}_{(X)}(-1)^{F} y^{F_{L}} q^{L_{0}-d / 8} \bar{q}^{\bar{L}_{0}-d / 8},
$$

where $\mathcal{H}(X)$ is the Hilbert space of the $\mathcal{N}=2$ superconformal field theory with target space $X$.

The formula (2.2) has the form of a trace over an irreducible representation of the Virasoro algebra containing a vacuum state $|0\rangle$ of a certain weight $k$, with $L_{0}|0\rangle=-k|0\rangle$, along with its Virasoro descendants $L_{-n_{1}} \cdots L_{-n_{l}}|0\rangle$. By the modular invariance of the path integral, the elliptic genus (2.2) is almost a modular form under the $S L(2, \mathbb{Z})$ transformations of $\tau \in \mathbb{C}^{+}$, and by the spectral flow it is almost an elliptic function under lattice translations of $z \in \mathbb{C}$ by $\mathbb{Z}+\mathbb{Z} \tau$. Unitarity implies that the elliptic genus is a weak Jacobi form such that the contribution to the trace from momentum modes of the bosonised $U(1)$ current can be separated into theta-functions which are the Jacobi forms of weight $1 / 2$ on the subgroups of $S L(2, \mathbb{Z})$.

The field theory elliptic genus (2.2) may be alternatively defined in terms of characteristic classes of $X$ [36, 19, 12]. For any complex vector bundle $V$ over $X$, we can define the invertible formal sums

$$
\bigwedge_{q} V=\bigoplus_{k=0}^{d} q^{k} \bigwedge^{k} V \quad \text { and } \quad \mathfrak{S}_{q} V=\bigoplus_{k=0}^{\infty} q^{k} \mathfrak{S}^{k} V
$$

where $\bigwedge^{k} V$ (resp. $\mathfrak{S}^{k} V$ ) denotes the $k$-th exterior (resp. symmetric) product of $V$. Introduce 
the invertible bundle

$$
\mathcal{E} l l(V ; q, y)=y^{-d / 2} \bigotimes_{n=1}^{\infty}\left(\bigwedge_{-y q^{n-1}} V \otimes \bigwedge_{-y^{-1} q^{n}} V^{*} \otimes \mathfrak{S}_{q^{n}} V \otimes \mathfrak{S}_{q^{n}} V^{*}\right)
$$

regarded as a formal power series in $(q, y)$ with coefficients in the complex K-theory group $K^{0}(X)$. 2 It is straightforward to see that the $\mathrm{K}$-theory operation $\mathcal{E} l l$ is multiplicative, in the sense that for any two complex vector bundles $V$ and $W$ on $X$ one has

$$
\begin{aligned}
& \mathcal{E} l l(V \oplus W ; q, y)=\mathcal{E} l l(V ; q, y) \otimes \mathcal{E} l l(W ; q, y) \\
& \mathcal{E} l l(V \ominus W ; q, y)=\mathcal{E} l l(V ; q, y) \otimes \mathcal{E} l l(W ; q, y)^{-1} .
\end{aligned}
$$

The formal power series

$$
\chi(X ; q, y):=\chi(X, \mathcal{E} l l(T X ; q, y))=\sum_{j=0}^{d}(-1)^{j} \operatorname{dim} H^{j}(X, \mathcal{E} l l(T X ; q, y))
$$

is a holomorphic function on $\mathbb{C}^{+} \times \mathbb{C}$, called the elliptic genus of $X$. By the GrothendieckRiemann-Roch theorem, it can be computed in terms of characteristic classes as

$$
\begin{aligned}
\chi(X ; q, y) & =\int_{X} \operatorname{ch}(\mathcal{E} l l(T X ; q, y)) \operatorname{Td}(X) \\
& =y^{-d / 2} \int_{X} \prod_{j=1}^{d} x_{j} \prod_{n=1}^{\infty}\left[\frac{\left(1-y q^{n-1} \mathrm{e}^{-x_{j}}\right)\left(1-y^{-1} q^{n} \mathrm{e}^{x_{j}}\right)}{\left(1-q^{n-1} \mathrm{e}^{-x_{j}}\right)\left(1-q^{n} \mathrm{e}^{x_{j}}\right)}\right],
\end{aligned}
$$

where $\left\{x_{j}\right\}_{j=1}^{d}$ are the Chern roots of the complex tangent bundle $T X$. For a Calabi-Yau manifold $X$ of dimension $d$, the elliptic genus (2.7) is a weak Jacobi form of weight zero and index $d / 2$ [36, 12].

For $q=0$ the elliptic genus (2.2) enumerates the BPS states with $L_{0}=\bar{L}_{0}=d / 8$; one has $\mathcal{E} l l(T X ; 0, y)=y^{-d / 2} \bigwedge_{-y}(T X)$ and the elliptic genus reduces to the Hirzebruch $\chi_{y^{-}}$ genus [43]:

$$
\begin{aligned}
\chi_{y}(X)=y^{d / 2} \chi(X ; 0, y) & =\sum_{j=0}^{d}(-y)^{j} \chi\left(X, \bigwedge^{j} T X\right) \\
& =\sum_{j=0}^{d}(-y)^{j} \sum_{k=0}^{d}(-1)^{k} \operatorname{dim} H^{k}\left(X, \Omega_{X}^{j}\right),
\end{aligned}
$$

so that

$$
\begin{aligned}
\chi(X ; q, y) & =y^{-d / 2} \chi_{y}(X)+q y^{-d / 2} \sum_{j=0}^{d}\left\{(-y)^{j+1} \chi\left(X, \bigwedge^{j} T X \otimes T X\right)\right. \\
& \left.+q(-y)^{j-1} \chi\left(X, \bigwedge^{j} T X \otimes T^{*} X\right)+q(-y)^{j} \chi\left(X, \bigwedge^{j} T X \otimes\left(T X \otimes T^{*} X\right)\right)\right\}+\ldots
\end{aligned}
$$

\footnotetext{
${ }^{2}$ Alternatively, we can regard $\mathcal{E} l l(V ; q, y)$ as a sheaf with a well-defined holomorphic Euler characteristic $\chi(X, \mathcal{E} l l(V ; q, y))=\sum_{j \geq 0}(-1)^{j} \operatorname{dim} \operatorname{Ext}^{j}\left(\mathcal{O}_{X}, \mathcal{E} l l(V ; q, y)\right)$.
} 
By the Grothendieck-Riemann-Roch theorem we have

$$
\chi_{y}(X)=\sum_{j=0}^{d}(-y)^{j} \int_{X} \operatorname{ch}\left(\Omega_{X}^{j}\right) \operatorname{Td}(X)=\int_{X} \prod_{j=1}^{d}\left[\frac{x_{j}\left(1-y \mathrm{e}^{-x_{j}}\right)}{\left(1-\mathrm{e}^{-x_{j}}\right)}\right] .
$$

For a Calabi-Yau manifold $X$ of dimension $d$, the $\chi_{y}$-genus transforms as

$$
\chi_{y}(X)=(-1)^{r-d} y^{r} \chi_{y^{-1}}(X)
$$

for some $r$. This relation can be derived from the Serre duality

$$
H^{j}\left(X, \bigwedge^{s} T X\right) \cong H^{d-j}\left(X, \bigwedge^{r-s} T X\right)
$$

Points. Let $X$ be a point. Then $V$ is a finite-dimensional complex vector space, which we assume to be $\mathbb{Z}$-graded. If $\mathcal{E} l l(V ; q, y)=\bigoplus_{k, l} y^{k} q^{l} \mathcal{E}_{k l}(V)$, then the bi-graded vector space $\mathcal{E} l l(V):=\bigoplus_{k, l} \mathcal{E}_{k l}(V)$ has the structure of an $\mathcal{N}=2$ superconformal vertex algebra.

Hypersurfaces. Let $X$ be a compact complex manifold of dimension $d$ and let $M \subset X$ be a smooth hypersurface. Denote by $[M]$ the line bundle on $X$ associated to the divisor $M$. The adjunction formula states that $\left.N_{M / X} \cong[M]\right|_{M}$, where $N_{M / X}=\left.T X\right|_{M} / T M$ is the normal bundle of $M$ in $X$. The exact sequence $\left.0 \rightarrow T M \rightarrow T X\right|_{M} \rightarrow N_{M / X} \rightarrow 0$ of bundles on $M$ can then be rewritten as

$$
\left.\left.0 \longrightarrow T M \longrightarrow T X\right|_{M} \longrightarrow[M]\right|_{M} \longrightarrow 0
$$

In K-theory, $T M=\left.(T X \ominus[M])\right|_{M}$ is the virtual tangent bundle of $M$ in $X$, and in terms of Chern roots $\left\{x_{j}\right\}_{j=1}^{d}$ we have 3

$$
\begin{aligned}
\chi(M ; q, y) & =y^{-(d-1) / 2} \int_{X} \prod_{n=1}^{\infty}\left[\frac{\left(1-q^{n-1} \mathrm{e}^{-c_{1}([M])}\right)\left(1-q^{n} \mathrm{e}^{c_{1}([M])}\right)}{\left(1-y q^{n-1} \mathrm{e}^{-c_{1}([M])}\right)\left(1-y^{-1} q^{n} \mathrm{e}^{c_{1}([M])}\right)}\right] \\
& \times \prod_{j=1}^{d} x_{j} \prod_{n=1}^{\infty}\left[\frac{\left(1-y q^{n-1} \mathrm{e}^{-x_{j}}\right)\left(1-y^{-1} q^{n} \mathrm{e}^{x_{j}}\right)}{\left(1-q^{n-1} \mathrm{e}^{-x_{j}}\right)\left(1-q^{n} \mathrm{e}^{x_{j}}\right)}\right] .
\end{aligned}
$$

For $q=0$ one has $\mathcal{E} l l([M] ; 0, y)=y^{-1 / 2}(1 \ominus y[-M])$ and according to [44],

$$
\chi_{y}(M)=\chi\left(X, \bigwedge_{-y} T X \otimes(1 \ominus y[-M])^{-1} \otimes(1 \ominus[-M])\right) .
$$

In terms of Chern roots, we finally obtain

$$
\chi_{y}(M)=\int_{X} \frac{\left(1-\mathrm{e}^{-c_{1}([M])}\right)}{\left(1-y \mathrm{e}^{-c_{1}([M])}\right)} \prod_{j=1}^{d}\left[\frac{x_{j}\left(1-y \mathrm{e}^{-x_{j}}\right)}{\left(1-\mathrm{e}^{-x_{j}}\right)}\right] .
$$

\footnotetext{
${ }^{3}$ Note that $c(M)=\left.c(X) c([M])^{-1}\right|_{M}$. Let $c(V)=\prod_{j} c\left(V_{j}\right)^{(-1)^{j}}$ for a $\mathbb{Z}$-graded vector bundle $V=\bigoplus_{j} V_{j}$. We have $c(M)=\left.c(T X \ominus[M])\right|_{M}$, and $\chi(M)=\int_{M} c(M)=\int_{X} c(M) c_{1}\left(N_{M / X}\right)=\int_{X} c(T X \ominus[M]) c_{1}([M])$, where $c_{1}([M])$ is the first Chern class of $[M]$.
} 
Complete intersections. Suppose now that $V \rightarrow X$ is a holomorphic vector bundle on $X$ of rank $r$. Let $s: X \rightarrow V$ be a holomorphic section transverse to the zero section such that the zero set $Y:=s^{-1}(0)$ is a complex submanifold of $X$, with $\left.N_{Y / X} \cong V\right|_{Y}$. From the exact sequence $\left.0 \rightarrow T Y \rightarrow T X\right|_{Y} \rightarrow N_{Y / X} \rightarrow 0$ and the multiplicative property of $\mathcal{E} l l$ it follows that 44

$$
\mathcal{E} l l(T Y ; q, y) \otimes \mathcal{E} l l\left(\left.V\right|_{Y} ; q, y\right)=\mathcal{E} l l\left(\left.T X\right|_{Y} ; q, y\right)=\left.\mathcal{E} l l(T X ; q, y)\right|_{Y}
$$

so that $\mathcal{E} l l(T Y ; q, y)=\left.\mathcal{E} l l(T X ; q, y) \otimes \mathcal{E} l l(V ; q ; y)^{-1}\right|_{Y}$ and hence

$$
\chi(Y, \mathcal{E} l l(T Y ; q, y))=\chi\left(Y,\left.\mathcal{E} l l(T X \ominus V ; q, y)\right|_{Y}\right) .
$$

By tensoring the Koszul complex [31]

$$
0 \longrightarrow \mathcal{O}_{X}\left(\bigwedge^{r} V^{*}\right) \longrightarrow \mathcal{O}_{X}\left(\bigwedge^{r-1} V^{*}\right) \longrightarrow \cdots \longrightarrow \mathcal{O}_{X}\left(\bigwedge^{1} V^{*}\right) \longrightarrow \mathcal{O}_{X} \longrightarrow \mathcal{O}_{Y} \longrightarrow 0
$$

with the bundle $\mathcal{E} l l(T X \ominus V ; q, y)$, we get the exact sequence

$$
\begin{aligned}
0 & \longrightarrow \mathcal{O}_{X}\left(\mathcal{E l l}(T X \ominus V ; q, y) \otimes \bigwedge^{r} V^{*}\right) \longrightarrow \mathcal{O}_{X}\left(\mathcal{E l l}(T X \ominus V ; q, y) \otimes \bigwedge^{r-1} V^{*}\right) \longrightarrow \cdots \\
& \longrightarrow \mathcal{O}_{X}\left(\mathcal{E l l}(T X \ominus V ; q, y) \otimes \bigwedge^{1} V^{*}\right) \longrightarrow \mathcal{O}_{X}(\mathcal{E l l}(T X \ominus V ; q, y)) \\
& \left.\longrightarrow \mathcal{O}_{X}(\mathcal{E} l l(T X \ominus V ; q, y))\right|_{Y} \longrightarrow 0 .
\end{aligned}
$$

Then by taking the Riemann-Roch character of this complex one gets [44]

$$
\chi(Y ; q, y)=\chi\left(Y,\left.\mathcal{E} l l(T X \ominus V ; q, y)\right|_{Y}\right)=\chi\left(X, \mathcal{E} l l(T X \ominus V ; q, y) \otimes \bigwedge_{-1} V^{*}\right) .
$$

Denoting by $\left\{v_{k}\right\}_{k=1}^{r}$ the Chern roots of $V$, we thus have

$$
\begin{aligned}
\chi(Y ; q, y) & =y^{-\operatorname{dim} Y / 2} \int_{X} \prod_{k=1}^{r} \prod_{n=1}^{\infty}\left[\frac{\left(1-q^{n-1} \mathrm{e}^{-v_{k}}\right)\left(1-q^{n} \mathrm{e}^{v_{k}}\right)}{\left(1-y q^{n-1} \mathrm{e}^{-v_{k}}\right)\left(1-y^{-1} q^{n} \mathrm{e}^{v_{k}}\right)}\right] \\
& \times \prod_{j=1}^{d} x_{j} \prod_{n=1}^{\infty}\left[\frac{\left(1-y q^{n-1} \mathrm{e}^{-x_{j}}\right)\left(1-y^{-1} q^{n} \mathrm{e}^{x_{j}}\right)}{\left(1-q^{n-1} \mathrm{e}^{-x_{j}}\right)\left(1-q^{n} \mathrm{e}^{x_{j}}\right)}\right] .
\end{aligned}
$$

Note that the last products in the integrands of (2.11) and (2.15) are the same, as both have been calculated in terms of Chern roots of the tangent bundle $T X$; the first product in the integrand of (2.15) is given in terms of Chern roots of the vector bundle $V \rightarrow X$. Setting $q=0$ we get back a similar result to (2.13) with

$$
\begin{aligned}
\chi_{y}(Y) & =\chi\left(X, \bigwedge_{-y} T X \otimes\left(\bigwedge_{-y} V^{*}\right)^{-1} \otimes \bigwedge_{-1} V^{*}\right) \\
& =\int_{X} \prod_{k=1}^{r}\left[\frac{\left(1-\mathrm{e}^{-v_{k}}\right)}{\left(1-y \mathrm{e}^{-v_{k}}\right)}\right] \prod_{j=1}^{d}\left[\frac{x_{j}\left(1-y \mathrm{e}^{-x_{j}}\right)}{\left(1-\mathrm{e}^{-x_{j}}\right)}\right] .
\end{aligned}
$$




\subsection{Superconformal sigma-models on symmetric products}

As shown in Ref. [19], the elliptic genus of an $\mathcal{N}=2$ superconformal sigma-model on a symmetric product orbifold $\mathfrak{S}^{n} X$ equates to the partition function of a second quantized string theory on a space $X$ with $S^{1}$-action. 4 In string compactifications on manifolds of the form $X \times S^{1}$, one can consider the configuration of a D-string wound $n$ times around $S^{1}$, and bound to a $\mathrm{D} p$-brane where $p=d+1$. The quantum mechanical degrees of freedom of this D-brane configuration are naturally encoded in a two-dimensional sigma-model on the $n$-th symmetric product of $X$, which describes the transverse fluctuations of the D-string; this construction is originally due to [53, 7, 51].

Let us therefore consider a sigma-model on the $n$-th symmetric product $\mathfrak{S}^{n} X$ of a Kähler manifold $X$ of dimension $d$, which is the orbispace

$$
\mathfrak{S}^{n} X=\left[X^{n} / \mathfrak{S}_{n}\right]:=\underbrace{X \times \cdots \times X}_{n} / \mathfrak{S}_{n},
$$

where $\mathfrak{S}_{n}$ is the symmetric group of order $n$ acting by permuting factors. Objects of the category of the orbifold stack $\left[X^{n} / \mathfrak{S}_{n}\right]$ are the $n$-tuples $\left(x_{1}, \ldots, x_{n}\right)$ of points in $X$; arrows are elements of the form $\left(x_{1}, \ldots, x_{n} ; \sigma\right)$, where $\sigma \in \mathfrak{S}_{n}$. The arrow $\left(x_{1}, \ldots, x_{n} ; \sigma\right)$ has source $\left(x_{1}, \ldots, x_{n}\right)$ and target $\left(x_{\sigma(1)}, \ldots, x_{\sigma(n)}\right)$. This category is a groupoid: the inverse of $\left(x_{1}, \ldots, x_{n} ; \sigma\right)$ is $\left(x_{\sigma(1)}, \ldots, x_{\sigma(n)} ; \sigma^{-1}\right)$. The genus one partition function depends on the boundary conditions imposed on the fermionic fields. For definiteness, following [19] we choose the boundary conditions such that the partition function $\chi\left(\mathfrak{S}^{n} X ; q, y\right)$ coincides with the elliptic genus defined as in (2.2).

Generating functions. The Hilbert space of an orbifold string theory can be decomposed into twisted sectors $\mathcal{H}_{\gamma}$ which are labelled by the conjugacy classes $\{\gamma\}$ of the orbifold group $\mathfrak{S}_{n}$. For a given twisted sector one keeps only those states that are invariant under the centralizer subgroup $G_{\gamma}$ of the element $\gamma$; let $\mathcal{H}_{\gamma}^{G_{\gamma}}$ be an invariant subspace associated with $G_{\gamma}$. One can parametrize the conjugacy classes $[\gamma]$ by using a set of partitions $\left\{n_{j}\right\}_{j=1}^{s(\gamma)}$, $\sum_{j=1}^{s(\gamma)} n_{j}=n$, where $n_{j}$ is the multiplicity of the cyclic permutation $(j)$ of $j$ elements in the decomposition of $\gamma:[\gamma]=\prod_{j=1}^{s(\gamma)}(j)^{n_{j}}$. For this conjugacy class the centralizer subgroup of a permutation $\gamma$ is 19

$$
G_{\gamma}=\mathfrak{S}_{n_{1}} \times \prod_{j=2}^{s(\gamma)}\left(\mathfrak{S}_{n_{j}} \rtimes \mathbb{Z}_{j}^{n_{j}}\right),
$$

where each subfactor $\mathfrak{S}_{n_{j}}$ and $\mathbb{Z}_{j}$ permutes the $n_{j}$ cycles $(j)$ and acts within each cycle $(j)$ respectively. The total orbifold Hilbert space $\mathcal{H}\left(\mathfrak{S}^{n} X\right)$ takes the form [19]

$$
\mathcal{H}\left(\mathfrak{S}^{n} X\right)=\bigoplus_{[\gamma]} \mathcal{H}_{\gamma}^{G_{\gamma}}=\bigoplus_{[\gamma]} \bigotimes_{j=1}^{s(\gamma)} \mathfrak{S}^{n_{j}} \mathcal{H}_{(j)}^{\mathbb{Z}_{j}},
$$

where $\mathfrak{S}^{n} \mathcal{H}:=\left(\mathcal{H}^{\otimes n}\right)^{\mathfrak{S}_{n}}$ and we have decomposed each twisted sector $\mathcal{H}_{\gamma}^{G_{\gamma}}$ into a product over the subfactors $(j)$ of $n_{j}$-fold symmetric tensor products.

\footnotetext{
${ }^{4}$ See [19, Sect. 1.1] for a string theory interpretation of superconformal sigma-models on $\mathfrak{S}^{n} X$.
} 
Let $\chi(-; q, y)$ be the generating function for any Hilbert (sub)space of a superconformal sigma-model; in [40] it is shown that the generating function for $\mathcal{H}\left(\mathfrak{S}^{n} X\right)$ coincides with the elliptic genus of $X$. If $\chi\left(\mathcal{H}_{(j)}^{\mathbb{Z}_{j}} ; q, y\right)$ admits the expansion $\chi\left(\mathcal{H}_{(j)}^{\mathbb{Z}_{j}} ; q, y\right)=\sum_{n \geq 0, l} \kappa(j n, l) q^{n} y^{l}$, then one has the product formula [36, 19]

$$
\sum_{n=0}^{\infty} p^{n} \chi\left(\mathfrak{S}^{n} X ; q, y\right)=\prod_{m>0, n \geq 0, l}\left(1-p^{m} q^{n} y^{l}\right)^{-\kappa(m n, l)},
$$

where $p:=\mathrm{e}^{2 \pi \mathrm{i} \rho}$ with $\rho$ the complexified Kähler form of $\mathcal{E}$. One can enhance the $S L(2, \mathbb{Z}) \times$ $S L(2, \mathbb{Z})$ transformations of the parameters $(\rho, \tau)$ together with the elliptic transformations of the Wilson line modulus $z$ by combining them into a $2 \times 2$ matrix

$$
\Xi=\left(\begin{array}{ll}
\rho & z \\
z & \tau
\end{array}\right)
$$

belonging to the Siegel upper half-plane of genus two, with $\operatorname{Im} \rho>0, \operatorname{Im} \tau>0$, and $\operatorname{det} \operatorname{Im} \Xi>0$. The Narain duality group $S O(3,2 ; \mathbb{Z})$ is isomorphic to the Siegel modular group $S p(4, \mathbb{Z})$ which acts on the matrix $\Xi$ by linear fractional transformations $\Xi \mapsto$ $(A \Xi+B)(C \Xi+D)^{-1}$. Then the elliptic genus is almost an automorphic form for $O(3,2 ; \mathbb{Z})$.

For $q=0$ the elliptic genus reduces to the $\chi_{y}$-genus of $\mathfrak{S}^{n} X$ and by (2.8) the generating series (2.19) becomes

$$
\sum_{n=0}^{\infty} q^{n} \chi_{y}\left(\mathfrak{S}^{n} X\right)=\prod_{j=0}^{d} \prod_{n=1}^{\infty}\left(1-y^{n+j} q^{n}\right)^{(-1)^{j+1} \chi^{j}(X)}
$$

where $\chi^{j}(X):=\sum_{k=0}^{d}(-1)^{k} h^{j, k}(X)$ and $h^{j, k}(X)$ are the Hodge numbers of $X$.

For trivial line bundles $y=1$, the elliptic genus of the symmetric product $\mathfrak{S}^{n} X$ degenerates to the Witten index of the supersymmetric field theory which is given by the Euler number $\chi\left(\mathfrak{S}^{n} X ; q, 1\right)=\chi\left(\mathfrak{S}^{n} X\right)$ [32, 52]. This identifies the elliptic genus with the character of the bosonic Fock module for the Heisenberg algebra, which is almost a modular form of weight $-\chi(X) / 2$, through the identity

$$
\sum_{n=0}^{\infty} q^{n} \chi\left(\mathfrak{S}^{n} X\right)=\hat{\eta}(\tau)^{-\chi(X)}=\prod_{n=1}^{\infty}\left(1-q^{n}\right)^{-\chi(X)},
$$

where $\hat{\eta}(\tau)$ is the Euler function which is related to the Dedekind function by $\eta(\tau):=$ $q^{1 / 24} \hat{\eta}(\tau)$. A similar formula, associated to the (equivariant) orbifold Euler characteristic of the symmetric product, can be derived using the $\mathfrak{S}_{n}$-equivariant K-theory of $X^{n}$ by means of the expression $\chi\left(\mathfrak{S}^{n} X\right)=\operatorname{rank} K^{0}\left(\mathfrak{S}^{n} X\right)-\operatorname{rank} K^{1}\left(\mathfrak{S}^{n} X\right)$.

\subsection{Hilbert schemes and representations of affine Lie algebras}

The moduli space of zero-dimensional subschemes of length $n$ in a nonsingular quasi-projective variety $X$ over $\mathbb{C}$ is called the Hilbert scheme of $n$ points on $X$, and is denoted by $X^{[n]}$; it 
parametrizes D0-branes in terms of ideal sheaves supported at points of $X$. A simple example of a zero-dimensional subscheme is a collection of distinct points; the length is equal to the number of points. When points collide, more complicated subschemes appear as these configurations appear in families: when two points collide, there are infinitely many nearby points, which form a pair consisting of a point $x \in X$ and a one-dimensional subspace of the tangent space $T_{x} X$. This marks the difference between $X^{[n]}$ and the $n$-th symmetric product $\mathfrak{S}^{n} X=\left[X^{n} / \mathfrak{S}_{n}\right]$ in which the information about the one-dimensional subspace is absent.

When $\operatorname{dim} X=1$, the Hilbert scheme $X^{[n]}$ is isomorphic to $\mathfrak{S}^{n} X \cong X^{n}$ under the HilbertChow morphism. For $\operatorname{dim} X=2$, the Hilbert-Chow morphism $\pi: X^{[n]} \rightarrow \mathfrak{S}^{n} X$ gives a resolution of singularities of the symmetric product $\mathfrak{S}^{n} X$ 21]; in particular, $X^{[n]}$ is a nonsingular quasi-projective variety of dimension $2 n$. This is in contrast with Hilbert schemes for $\operatorname{dim} X>2$. For a projective variety $X$ the scheme $X^{[n]}$ is also projective, as follows from Grothendieck's construction of Hilbert schemes. Moreover, $X^{[n]}$ has a holomorphic symplectic form whenever $X$ has one [23, 2].

Some interesting non-compact examples include $X=\mathbb{C}^{2}$ and $X=T^{*} \Sigma$, where $\Sigma$ is a Riemann surface. For these complex surfaces there exists a $\mathbb{C}^{*}$-action on $X$ which naturally induces an action on $X^{[n]}[46]$. On any smooth complex surface $X$ one can construct a representation of products of Heisenberg and Clifford algebras on the direct sum of homology groups of all components $\bigoplus_{n>0} H_{\bullet}\left(X^{[n]}, \mathbb{C}\right)$; thus, the generating function can be interpreted as a character of the Heisenberg algebra. In particular, when $X$ is a Kähler manifold, the space $\bigoplus_{n \geq 0} H^{\bullet}\left(X^{[n]}, \mathbb{C}\right)$ forms a representation of the Heisenberg algebra generated by geometrically defined sigma-model creation and annihilation operators $\alpha_{n}^{a}$, where $a$ labels a basis of the cohomology $H^{\bullet}(X, \mathbb{C})$.

The relations between $\mathfrak{S}^{n} X$ and $X^{[n]}$ bear many similarities to the relations between string theory and field theory. For example, the Montonen-Olive S-duality conjecture implies that the generating function for the Euler numbers of moduli spaces of instantons in four dimensions should have modular properties. For $X$ a K3 surface, the Euler numbers of the moduli spaces of instantons are the same as those of Hilbert schemes of points on $X$. Strictly speaking, one should consider moduli spaces of stable sheaves instead of moduli spaces of instantons which are usually non-compact, and this point of view will be taken in what follows. Then Göttsche's formula (2.22) below gives the desired answer. Moreover, the homology groups of moduli spaces of instantons on an ALE space, i.e. the minimal resolution of a simple Kleinian singularity, form an integrable representation of a Kac-Moody algebra [46]. The modular properties of the characters of the representation (equivalently string partition functions) can be described within the framework of conformal field theory. Heisenberg algebras and other affine Lie algebras could perhaps be understood in this way within the framework of heterotic/Type IIA duality.

Göttsche's formula. When $X$ is an algebraic surface, the formula (2.21) also computes the topological Euler characteristic of the Hilbert scheme $X^{[n]}$ [29], while (2.19) computes the elliptic genus of $X^{[n]}$ [13]. More generally, the generating function of the Poincaré 
polynomials $P_{t}\left(X^{[n]}\right)=\sum_{j=0}^{4} t^{j} b_{j}\left(X^{[n]}\right)$ of $X^{[n]}$ is given by the product formula

$$
\sum_{n=0}^{\infty} q^{n} P_{t}\left(X^{[n]}\right)=\prod_{j=0}^{4} \prod_{n=1}^{\infty}\left(1-(-t)^{2 n+j-2} q^{n}\right)^{(-1)^{j+1} b_{j}(X)}
$$

where $b_{j}(X)=\operatorname{dim} H_{j}(X, \mathbb{R})$ is the degree $j$ Betti number of $X$.

When $X$ is a smooth quasi-projective Calabi-Yau threefold, the Hilbert scheme $X^{[n]}$ is generally not smooth and has a complicated subscheme structure with branches of varying dimension. Nevertheless, in this case it is still possible to define virtual cycles and a virtual Euler characteristic $\chi^{\mathrm{vir}}\left(X^{[n]}\right)$, which computes the degree zero Donaldson-Thomas invariants of $X$, enumerating D0-branes parametrized by ideal sheaves supported at points of $X$. Then there is an analog of Göttsche's formula which reads as [3]

$$
\sum_{n=0}^{\infty} q^{n} \chi^{\mathrm{vir}}\left(X^{[n]}\right)=M(\tau)^{\chi(X)}=\prod_{n=1}^{\infty}\left(1-q^{n}\right)^{-n \chi(X)}
$$

where $M(\tau)$ is the MacMahon function which can be represented as a certain vacuum correlation function of vertex operators for the Heisenberg algebra. More generally, the generating function for the virtual version of the Poincaré polynomials $P_{t}^{\mathrm{vir}}\left(X^{[n]}\right)$ of the Hilbert scheme $X^{[n]}$ is the analog of (2.22) given by [4]:

$$
\sum_{n=0}^{\infty} q^{n} P_{t}^{\mathrm{vir}}\left(X^{[n]}\right)=\prod_{j=0}^{6} \prod_{n=1}^{\infty} \prod_{m=0}^{n-1}\left(1-(-t)^{2 m-n+j-2} q^{n}\right)^{(-1)^{j+1} b_{j}(X)} .
$$

\section{M-theory partition functions and $(4,0)$ elliptic gen- era}

\subsection{Black hole partition functions in the M-theory frame}

If $X$ is a nonsingular compact Calabi-Yau threefold, then four-dimensional BPS black holes in M-theory compactifications on $X \times S^{1}$ can be represented microscopically by wrapping an M5-brane with fluxes and Kaluza-Klein momentum on $M \times S^{1}$, where $M$ is a divisor in $X$. The dimensionally reduced M5-brane worldvolume theory is dual to the Ramond sector of a $(4,0)$ superconformal field theory [45]; the Neveu-Schwarz sector of this conformal field theory is holographically dual to 11-dimensional supergravity (and the five-dimensional black ring [5]) on the attractor geometry $A d S_{3} \times S^{2} \times X$. The black hole microstates are represented by the supersymmetric ground states of this $(4,0) C F T_{2}$ which are counted by an appropriately defined elliptic genus describing the BPS gravitational configurations inside $A d S_{3}$.

The black holes in question can be viewed as excitations of black strings, whose near-horizon geometry is a three-dimensional anti-de Sitter space $A d S_{3}$. The Euclidean thermal $A d S_{3}$ has a conformal elliptic curve $\mathcal{E}$ as its boundary whose complex structure modulus is $\tau$. 
The partition function of the dual boundary conformal field theory $C F T_{2}$ depends on $\tau$. The black hole partition functions are then expected to possess a modular symmetry which is identified with the mapping class group $S L(2, \mathbb{Z})$ of the boundary elliptic curve; after a choice of line bundle on $\mathcal{E}$, they are also expected to possess an elliptic symmetry which is identified with large gauge transformations of the three-form field $H$ that leads to a spectral flow symmetry of the partition function for the black string.

Consider the two-dimensional $(4,0)$ superconformal field theory on the boundary $\mathcal{E}$ of $A d S_{3}$ which describes M5-branes wrapping compact four-cycles in $X$ [45]; we consider $\mathcal{E}$ to be a product of two circles $S^{1} \times S_{m}^{1}$. We are interested in charge configurations consisting of M5M2 brane bound states which carry momentum $q_{0}$ along $S_{m}^{1}$, with a single M5-brane wrapping $M \times S_{m}^{1}$, where $M$ is a very ample divisor in $X$, and M2-branes wrapping holomorphic curves $\mathcal{C}$ in $M$. The divisor $M$ has a cohomology class $c_{1}([M]) \in H^{2}(X, \mathbb{Z})$, which can be expanded as $c_{1}([M])=p^{a} \Sigma_{a}$, where $\Sigma_{a}$ is a basis for $H^{2}(X, \mathbb{Z}) 5$ Via the pullback of the embedding $M \subset X$ this induces a basis for $H^{2}(M, \mathbb{Z})$ which for simplicity we also denote by $\Sigma_{a}$. Then the M2-brane charges $q_{a}$ are given by expanding $\mathcal{C}=q_{a} \mathcal{C}^{a}$ where $\mathcal{C}^{a}$ are dual two-cycles to $\Sigma_{a}$ in the lattice $\Gamma:=H_{2}(M, \mathbb{Z})$. They correspond to the numbers of left-moving excitations of total momentum $q_{0}$ on the M5-brane. In the basis $\Sigma_{a}$ the triple intersection form of $X$ is

$$
D_{a b c}=\frac{1}{6} \int_{X} \Sigma_{a} \wedge \Sigma_{b} \wedge \Sigma_{c},
$$

while the intersection matrix of $M$ is given by

$$
C_{a b}=-\int_{M} \Sigma_{a} \wedge \Sigma_{b}=-6 D_{a b}, \quad \text { with } \quad D_{a b}:=D_{a b c} p^{c} .
$$

We denote the inverse matrices to $C_{a b}, D_{a b}$ by $C^{a b}, D^{a b}$. Let $b_{2}^{ \pm}(M)$ denote the dimensions of the spaces of selfdual/anti-selfdual two-forms on $M$. Then there are $b_{2}^{+}(M)$ right-moving scalars on $S_{m}^{1}$ from dimensional reduction of the self-dual three-form field strength $H$, and by the Riemann-Roch formula one has

$$
b_{2}^{+}(M)=2 D+\frac{1}{6}\left(c_{2}\right)_{a} p^{a}
$$

where $D=D_{a b c} p^{a} p^{b} p^{c}$ and $\left(c_{2}\right)_{a}=\int_{X} \Sigma_{a} \wedge c_{2}(X)$. The Euler characteristic of the divisor $M$ is also computed by the Riemann-Roch theorem as

$$
\chi(M)=2+b_{2}^{+}(M)+b_{2}^{-}(M)=\int_{M} c_{2}(M)=6 D+\left(c_{2}\right)_{a} p^{a}=c_{L},
$$

which coincides with the central charge $c_{L}$ of the conformal field theory, i.e. the number of left-moving chiral bosons in the M-theory frame.

The black hole partition function in the canonical ensemble is defined by

$$
\mathcal{Z}_{X}(p, \phi)=\sum_{q_{0}, q_{a}} d(q, p) \mathrm{e}^{-q_{0} \phi_{0}-q_{a} \phi^{a}},
$$

\footnotetext{
${ }^{5}$ In this paper all integer cohomology groups are understood modulo their torsion subgroups.
} 
where the quantum degeneracy $d(q, p)$ of black holes is the Witten index

$$
d(q, p)=\operatorname{Tr}_{\mathcal{H}_{q, p}(X)}(-1)^{F}
$$

computed in the Ramond sector of the Hilbert space $\mathcal{H}_{q, p}(X)$ of the underlying $(4,0)$ superconformal field theory with target space $X$. Here $\phi_{0}$ and $\phi^{a}$ are the respective chemical potentials conjugate to the M-momentum and membrane charges. Being an index, it receives only contributions from BPS states and is independent of the background moduli. Once the black hole partition function is known, the index of BPS states may be recovered from a suitable attractor contour integral prescription:

$$
d(q, p)=\int_{C} \mathrm{~d} \phi_{0} \prod_{a} \mathrm{~d} \phi^{a} \mathrm{e}^{q_{0} \phi_{0}+q_{a} \phi^{a}} \mathcal{Z}_{X}(p, \phi)
$$

and the leading semi-classical approximation $\mathrm{e}^{S(q, p)}$ yields the Bekenstein-Hawking-Wald entropy $S(q, p)$. For the partition functions we compute in the following, the singularity and pole structures required to evaluate such contour integrals are described in Appendix B.

Later on we shall also consider black hole entropy counting on certain classes of local CalabiYau backgrounds $X$. Such non-compact Calabi-Yau manifolds can sometimes be thought of as decompactification limits of compact Calabi-Yau threefolds in neighbourhoods of collapsing cycles. In this limit, gravity decouples so one needs to be careful about what is meant by a "black hole" partition function. In the following, it will always mean that the branes which microscopically realise the BPS black hole wrap a non-compact cycle in the limit with nonzero entropy - a BPS "black hole" is thus a BPS particle with large entropy. On non-compact Calabi-Yau manifolds $X$, a natural refinement of the quantum black hole degeneracies (3.6) can be defined by replacing the Witten index with a protected spin character that depends on an extra parameter $y$ which gives a chemical potential conjugate to the angular momentum of states carrying the $S U(2)$ R-symmetry that appears in the gravity decoupling limit [1]. The protected spin character is a supersymmetric index in M-theory, hence it also receives contributions only from BPS black holes, and it is defined by

$$
d_{y}(q, p)=\operatorname{Tr}_{\mathcal{H}_{q, p}(X)}(-1)^{2 J_{3}} y^{J_{3}-J_{0}}
$$

where $J_{3}$ is the generator of the $\operatorname{Spin}(3)$ rotation group and $J_{0}$ is the left-moving $U(1)$ R-charge operator of the four-dimensional BPS states. This refined index counts spinning M2-branes wrapping holomorphic curves $\mathcal{C}$ in $X$, and the refined black hole partition function is a two-variable generalization of (3.5) defined by

$$
\mathcal{Z}_{X}(p, \phi, y)=\sum_{q_{0}, q_{a}} d_{y}(q, p) \mathrm{e}^{-q_{0} \phi_{0}-q_{a} \phi^{a}} .
$$

\subsection{Quantum gravity and spectral partition functions on $\operatorname{Ad} S_{3}$}

In the light of the $A d S_{3} / C F T_{2}$ correspondence, we expect a duality between spectral zetafunctions of Euclidean $A d S_{3}$ and elliptic modular forms. In particular, we consider spectral 
functions of hyperbolic three-geometry whose arguments take values on the elliptic curve $\mathcal{E}$, which is the conformal boundary of $A d S_{3}$, and are related to the congruence subgroup of the modular group $S L(2, \mathbb{Z})$ which fixes one of the spin structures on $\mathcal{E}$; they are connected to various modular-like forms (in particular Poincaré series) and thereby provide a spacetime perspective on the link between quantum gravity partition functions and characters of conformal field theory. The simplest example is provided by the Fock module character $\hat{\eta}(\tau)$ from Sect. 2.2 which can be expressed in terms of Selberg-type spectral Ruelle functions (see Appendix (A) as

$$
\hat{\eta}(\tau)=\mathcal{R}(s=1-\mathrm{i} \varrho(\tau)),
$$

where $\varrho(\tau)=\operatorname{Re} \tau / \operatorname{Im} \tau$. These spectral functions carry information about the holographically dual three-dimensional field theory, as their zeroes coincide with the scattering resonance poles of the Laplace operator on the hyperbolic three-manifold.

In the following, we will describe the roles of modular forms and spectral functions in black hole entropy counting. We will see that our black hole partition functions, in the guise of elliptic genera that compute one-loop supergravity partition functions, can be expressed in terms of spectral functions that provide spectral flow and a kind of modular invariance. Of particular relevance is the fact that the field theory elliptic genus (2.7) can be written in terms of the spectral Ruelle function $\mathcal{R}(s)$ as

$$
\begin{aligned}
\chi(X ; q, y) & =y^{-d / 2} \int_{X} \prod_{j=1}^{d} x_{j}\left[\frac{\mathcal{R}\left(\widehat{s}=\left(2 \pi \mathrm{i} z-x_{j}\right)(1-\mathrm{i} \varrho(\tau))\right)}{\mathcal{R}\left(\widehat{s}=-x_{j}(1-\mathrm{i} \varrho(\tau))\right)}\right] \\
& \times\left[\frac{\mathcal{R}\left(\widehat{s}=\left(2 \pi \mathrm{i}(\tau-z)+x_{j}\right)(1-\mathrm{i} \varrho(\tau))\right)}{\mathcal{R}\left(\widehat{s}=\left(2 \pi \mathrm{i} \tau+x_{j}\right)(1-\mathrm{i} \varrho(\tau))\right)}\right],
\end{aligned}
$$

where $q=\mathrm{e}^{2 \pi \mathrm{i} \tau}, y=\mathrm{e}^{2 \pi \mathrm{i} z}$ and $\widehat{s}=2 \pi \mathrm{i} \tau s$. Proceeding in this way, we shall find that our elliptic genera can be reproduced in terms of Selberg-type spectral functions of the asymptotic $A d S_{3}$ geometry, and thereby inherit the modular properties of the characters of highest-weight representations of the affine Lie algebras which underlie the holographically dual conformal field theories.

The role of elliptic modular forms and spectral functions of hyperbolic geometry can also be seen directly at the level of quantum corrections to gravity on $A d S_{3}$. One-loop corrections to three-dimensional gravity in locally anti-de Sitter spacetimes are qualitatively similar to black hole quantum corrections. The simple geometric structure of three-dimensional gravity allows exact computations, since its Euclidean counterpart is locally isometric to a constant curvature hyperbolic space $\mathbb{H}^{3}$. Then the quantum corrections to the gravity partition function on $A d S_{3}$ can be rewritten in terms of spectral Ruelle functions as [10, 11, 15

$$
\mathcal{Z}_{A d S_{3}}(\tau, \bar{\tau})=\prod_{n=2}^{\infty}\left|1-q^{n}\right|^{-2}=|\mathcal{R}(s=2-2 \mathrm{i} \varrho(\tau))|^{-2} .
$$

In the physics literature it is usually assumed that the fundamental domain for the action of a discrete isometry group $\mathfrak{G}^{\gamma}$ on three-dimensional hyperbolic spacetimes has finite volume. In contrast, a three-dimensional black hole has a Euclidean quotient representation $\mathbb{H}^{3} / \mathfrak{G}^{\gamma}$ 
for appropriate $\mathfrak{G}^{\gamma}$, where the fundamental domain has infinite hyperbolic volume; for the non-rotating black hole one can choose $\mathfrak{G}^{\gamma}$ to be the abelian group generated by a single hyperbolic element [50]. For the discrete groups of isometries of three-dimensional hyperbolic space with fundamental domain of infinite volume (e.g. for Kleinian groups, but excluding fundamental domains with cusps), Selberg-type functions and trace formulas are considered in [50]. In an infinite-volume setting the situation is complicated by the continuous spectrum of the Laplacian, and the absence of a canonical renormalization of the scattering operator for the associated Schrödinger equation which is required to render it trace-class. However, for a three-dimensional black hole one can bypass much of the general theory and proceed more directly, by defining a Selberg zeta-function attached to $\mathbb{H}^{3} / \mathfrak{G}^{\gamma}$ and establishing a trace formula which is a version of the Poisson resummation formula for resonances. In fact, there is a special relation between the spectrum and the truncated heat kernel of the Euclidean black hole with the Patterson-Selberg spectral function [14].

\subsection{Supergravity boson-fermion correspondence}

Let us consider in more detail the Neveu-Schwarz sector of the $(4,0)$ superconformal field theory on the $A d S_{3}$ boundary of the M-theory compactification on $A d S_{3} \times S^{2} \times X$. We shall compute the contribution to the elliptic genus of the $(4,0) C F T_{2}$ from supergravity states; they can be obtained, for example, from the fluctuation spectrum of 11-dimensional supergravity compactified on $A d S_{3} \times S^{2} \times X$. Later on we shall incorporate the contributions from wrapped M2-branes and M5-branes with fluxes and momentum on cycles in $A d S_{3} \times S^{2} \times$ $X$. We shall make explicit reference to the two left and right chiralities of the $C F T_{2}$, with the convention that they correspond respectively to the holomorphic and anti-holomorphic sectors. In addition to the underlying Virasoro algebras, we pay close attention to the $U(1)$ and R-symmetry current algebras.

To define the elliptic genus, we introduce chemical potentials conjugate to the charges $q_{a}, \bar{q}_{a}$ as before; the R-charge is $q_{0}$. Up to a spectral flow, supergravity states carry vanishing charges $q_{a}=0$; these charges are instead carried by wrapped M2-branes, which we consider below. Hence the contribution to the polar part of the elliptic genus from such supergravity states has the schematic form $\chi_{\text {sugra }}(q)=\sum_{n} C(n) q^{n}$ with $n=q_{0}$ and $q=\mathrm{e}^{-\phi_{0}}=: \mathrm{e}^{2 \pi \mathrm{i} \tau}$, and in order to extract the quantum degeneracies $C(n)$ we have to compute the genus contribution $\chi_{\text {sugra }}(q)$.

We work in the dilute gas expansion in which the contributions to the elliptic genus are dominated by multi-particle chiral primary states of massless supergravity modes of M-theory compactified on $X$, i.e. supersymmetric ground states of BPS hypermultiplets on $A d S_{3} \times S^{2}$. Their spectrum on $A d S_{3} \times S^{2}$ organises into short representations of $S L(2, \mathbb{R}) \times S U(1,1 \mid 2)$. Consider the finite-dimensional subalgebra of the minimal $\mathcal{N}=4$ superconformal algebra in the Neveu-Schwarz sector spanned by the operators $\left\{L_{0}, L_{ \pm 1}, T_{0}^{i}, G_{ \pm 1 / 2}^{i}, \bar{G}_{ \pm 1 / 2}^{i}\right\}_{i=1,2,3}$. Then a state $|\psi\rangle$ is said to be a chiral primary if $G_{-1 / 2}^{2}|\psi\rangle=\bar{G}_{-1 / 2}^{1}|\psi\rangle=0$ and $G_{n+1 / 2}^{a}|\psi\rangle=$ $\bar{G}_{n+1 / 2}^{a}|\psi\rangle=0$, for $n \geq 0$ and $a=1,2$. The subspace of chiral primary states is denoted $\mathcal{H}^{\prime}(X)$. 
The Neveu-Schwarz sector elliptic genus is related to that of the Ramond sector by spectral flow [39]; the contribution from supergravity states can be written as

$$
\chi_{\text {sugra }}^{\mathrm{NS}}(q)=\operatorname{Tr}_{\mathcal{H}^{\prime}(X)}(-1)^{\bar{q}_{0}} q^{L_{0}} .
$$

The elliptic genus receives contributions from right-moving chiral primary states obeying $\bar{h}=\frac{1}{2} \bar{q}_{0}$ [39], where $\bar{h}$ is the eigenvalue of Virasoro operator $\bar{L}_{0}$.

Consider the single particle spectrum, and suppose that it starts at a conformal weight $h_{\text {min }}=\bar{h}_{\text {min }}+s$ for some $s \sqrt[6]{6}$ For the bosonic contributions we compute

$$
\prod_{l, p=0}^{\infty} \sum_{m=0}^{\infty} q^{m\left(h_{\min }+l+p\right)}=\prod_{l, p=0}^{\infty}\left(1-q^{\bar{h}_{\min }+s+l+p}\right)^{-1},
$$

where $m$ labels the number of particles, $p$ labels the actions of the operators $\left(L_{-1}\right)^{p}$ to produce Virasoro descendant states, and $l$ labels the shift in conformal dimension $\bar{h}=\bar{h}_{\text {min }}+l$. Setting $n=l+p+1$ and using Eq. (A.9) from Appendix A for the boson and fermion contributions, we then get

$$
\begin{gathered}
\chi_{\text {sugra }}^{\mathrm{NS}, \text { bos }}(q)=\prod_{n=1}^{\infty}\left(1-q^{h_{\min }-1+n}\right)^{-n}=\prod_{n=1}^{\infty}\left[\mathcal{R}\left(s=\left(n+h_{\min }-1\right)(1-\mathrm{i} \varrho(\tau))\right)\right]^{-1}, \\
\chi_{\text {sugra }}^{\mathrm{NS}, f e r m}(q)=\prod_{n=1}^{\infty}\left(1-q^{h_{\min }-1+n}\right)^{n}=\prod_{n=1}^{\infty} \mathcal{R}\left(s=\left(n+h_{\min }-1\right)(1-\mathrm{i} \varrho(\tau))\right) .
\end{gathered}
$$

In the case of five-dimensional supergravity obtained by compactifying M-theory on the Calabi-Yau threefold $X$, the five-dimensional massless spectrum can be described in the $\mathcal{N}=2$ superfield formalism in terms of the numbers of vector multiplets $n_{V}=h^{1,1}(X)-1$, hypermultiplets $n_{H}=2\left(h^{2,1}(X)+1\right)$, and gravitino multiplets $n_{S}$, in addition to the graviton multiplet; the Hodge numbers $h^{j, k}(X)$ in this context are the numbers of generators of degree $(j, k)$ [8]. From Eqs. (3.10), (3.12) and (3.15) we then find the supergravity elliptic genus to be

$$
\begin{aligned}
\chi_{\text {sugra }}^{\mathrm{NS}}(q) & =\mathcal{R}(s=2(1-\mathrm{i} \varrho(\tau)))[\mathcal{R}(s=1-\mathrm{i} \varrho(\tau))]^{\mathfrak{N}\left(n_{V}, n_{H}, n_{S}\right)} \\
& \times \prod_{n=1}^{\infty}[\mathcal{R}(s=(n+1)(1-\mathrm{i} \varrho(\tau)))]^{-\chi(X)}
\end{aligned}
$$

where the number $\mathfrak{N}\left(n_{V}, n_{H}, n_{S}\right)$ depends on the multiplets and $\chi(X)=2\left(h^{1,1}(X)-h^{2,1}(X)\right)$ is the Euler number of the Calabi-Yau manifold $X$.

\subsection{Supergravity elliptic genus on $A d S_{3} \times S^{2} \times X$}

We shall now compute the full supergravity elliptic genus from M-theory following Ref. [26]. In the Neveu-Schwarz sector it is given by

$$
\mathcal{Z}_{\text {sugra }}(\tau, \phi)=\operatorname{Tr} \bar{L}_{0=J_{R}^{3}}(-1)^{F} q^{L_{0}} \mathrm{e}^{-q_{a} \phi^{a}} .
$$

\footnotetext{
6 The appropriate complete spectrum of single particle primary states can be found in e.g. 39. Note that multiparticle chiral primaries can be obtained by taking arbitrary tensor products of single particle chiral primary states.
} 
In this formula we have dropped a factor $q^{-c_{L} / 24}$ which corresponds to the ground state energy of $A d S_{3}$, and the $L_{0}$ contributions entering here are due entirely to wrapped membranes. There are two kinds of contributions: one from wrapped membranes and one from supergravity modes. For M2-branes wrapping a holomorphic curve $\mathcal{C}$ in $X$, the total spin of highest weight states arising from a hypermultiplet in the representation $\left(j_{L}, j_{R}\right)$ of the five-dimensional little group $S O(4)=S U(2)_{L} \times S U(2)_{R}$ is $J^{3}=\frac{1}{2} q_{a} p^{a}+m_{R}+m_{L}+\ell+\frac{1}{2}$, where $-j_{L, R} \leq m_{L, R} \leq j_{L, R}$ and $\ell \geq 0$ is the orbital angular momentum on $S^{2}$; the range of $j_{L}$ is determined by the genus of $\mathcal{C}$, while $j_{R}$ is related to the weight of the Lefschetz action on the deformation moduli space of $\mathcal{C}$, and $q_{a} p^{a}$ is the contribution from the quantization of the self-dual three-form field.

Let us first consider the contribution of membranes wrapping an isolated rational genus zero curve with fixed charges $\left(q_{a}\right) \in \Gamma^{*}:=\Gamma \backslash\{0\}$ and degeneracy $N_{\left(q_{a}\right)}$. In this case there is no internal $\left(j_{L}, j_{R}\right)$ angular momentum contribution, and we have

$$
\mathcal{Z}_{\text {sugra }}^{(0,0)}(\tau, \phi)=\prod_{p^{a}, n>0}\left[\left(1-q^{n+\frac{1}{2} q_{a} p^{a}} \mathrm{e}^{-q_{a} \phi^{a}}\right)\left(1-q^{n+\frac{1}{2} q_{a} p^{a}} \mathrm{e}^{q_{a} \phi^{a}}\right)\right]^{n N_{\left(q_{a}\right)}} .
$$

For higher genus curves $\mathcal{C}$ and general angular momenta $\left(j_{L}, j_{R}\right)$ we have

$$
\begin{aligned}
& \mathcal{Z}_{\text {sugra }}^{\left(j_{L}, j_{R}\right)}(\tau, \phi)= \prod_{\substack{p^{a}, n>0 \\
-j_{L} \leq m_{L} \leq j_{L}}}\left[\left(1-q^{n+\frac{1}{2} q_{a} p^{a}+2 m_{L}} \mathrm{e}^{-q_{a} \phi^{a}}\right)\right. \\
&\left.\times\left(1-q^{n+\frac{1}{2} q_{a} p^{a}+2 m_{L}} \mathrm{e}^{q_{a} \phi^{a}}\right)\right]^{(-1)^{2 j_{R}+2 j_{L}} n\left(2 j_{R}+1\right) N_{\left(q_{a}\right), j_{L}, j_{R}}},
\end{aligned}
$$

where the quantum degeneracies $N_{\left(q_{a}\right), j_{L}, j_{R}}$ of the spinning M2-branes are related to the Gopakumar-Vafa BPS invariants of $X$, as we discuss in more detail below. Finally, the net contribution from massless neutral supergravity modes including singletons is given [26] by $\hat{\eta}(\tau) M(\tau)^{\chi(X)}$, analogously to (3.16). By the Göttsche product formulas from Sect. 2.3. this shows that these modes are essentially parametrized by the punctual Hilbert schemes $X^{[n]}$ and are hence enumerated by the degree zero Donaldson-Thomas invariants of $X$.

The Legendre transform of our original black hole partition function (3.5) is computed by the elliptic genus of the $(4,0)$ superconformal field theory which is given as a Ramond sector trace

$$
\mathcal{Z}_{X}(\tau, \phi)=\operatorname{Tr}_{\mathcal{H}(X)}(-1)^{F} q^{L_{0}-c_{L} / 24} \bar{q}^{\bar{L}_{0}-c_{L} / 24} \mathrm{e}^{-q_{a} \phi^{a}} .
$$

Collecting all contributions together, in the dilute gas expansion around $\operatorname{Im} \tau \rightarrow \infty$ one gets $\mathcal{Z}_{X}(\tau, \phi)=q^{-c_{L} / 24} \mathcal{Z}_{\text {sugra }}(\tau, \phi)$ and hence

$$
\begin{aligned}
\mathcal{Z}_{X}(\tau, \phi) & =q^{-c_{L} / 24} \hat{\eta}(\tau) M(\tau)^{\chi(X)} \prod_{\substack{\left(q_{a}\right) \in \Gamma^{*} \\
p^{a}, n>0}} \prod_{\substack{2 j_{L}, R \geq 0 \\
-j_{L} \leq m_{L} \leq j_{L}}}\left[\left(1-q^{n+\frac{1}{2} q_{a} p^{a}+2 m_{L}} \mathrm{e}^{-q_{a} \phi^{a}}\right)\right. \\
& \left.\times\left(1-q^{n+\frac{1}{2} q_{a} p^{a}+2 m_{L}} \mathrm{e}^{q_{a} \phi^{a}}\right)\right]^{(-1)^{2 j_{R}+2 j_{L}} n\left(2 j_{R}+1\right) N_{(q a), j_{L}, j_{R}}}
\end{aligned}
$$


Using Eq. (A.9) from Appendix $\mathrm{A}$ we find the spectral function representation

$$
\begin{aligned}
\mathcal{Z}_{X}(\tau, \phi) & =q^{-c_{L} / 24} \mathcal{R}(s=1-\mathrm{i} \varrho(\tau)) \prod_{n=1}^{\infty}\left[[\mathcal{R}(s=n(1-\mathrm{i} \varrho(\tau)))]^{-\chi(X)}\right. \\
& \times \prod_{\left(q_{a}\right) \in \Gamma^{*}, p^{a}>0} \prod_{\substack{2 j_{L, R} \geq 0 \\
-j_{L} \leq m_{L} \leq j_{L}}}\left[\mathcal{R}\left(s=\left(n+q_{a}\left(\frac{p^{a}}{2}-\widehat{\phi}^{a}\right)+2 m_{L}\right)(1-\mathrm{i} \varrho(\tau))\right)\right. \\
& \left.\times \mathcal{R}\left(s=\left(n+q_{a}\left(\frac{p^{a}}{2}+\widehat{\phi}^{a}\right)+2 m_{L}\right)(1-\mathrm{i} \varrho(\tau))\right)\right]^{\left.(-1)^{2 j_{R}+2 j_{L}}\left(2 j_{R}+1\right) N_{(q a), j_{L}, j_{R}}\right]},(3 .)
\end{aligned}
$$

where $\widehat{\phi}^{a}:=\phi^{a} / 2 \pi \mathrm{i} \tau$.

\subsection{Spectral partition functions for BPS invariants}

We shall now explain how to rewrite our M-theory black hole partition functions geometrically in terms of BPS invariants for some local Calabi-Yau geometries $X$, and derive a spectral function formulation for it. This can be done by using the Gopakumar-Vafa conjecture [28] which rewrites the topological string partition function of $X$ as a sum over BPS configurations of M2-branes wrapping holomorphic curves $\mathcal{C}$ in $X$ and the M-circle. In contrast to the Gromov-Witten invariants of $X$ which are in general rational numbers, the generating series in M-theory of these invariants in all non-zero degrees and all genera has a particular form determined by certain integer invariants. There have been various proposals for the proof of this conjecture (see e.g. [35, 34, 43]); for irreducible curve classes $\mathcal{C}$ the GopakumarVafa invariants coincide with the Pandharipande-Thomas stable pair invariants [49] which enumerate D2-branes parametrized by stable sheaves supported on $\mathcal{C}$.

Let $\mathcal{F}_{X}^{\text {top }}(\tau, Q)$ be the generating series of Gromov-Witten invariants of a Calabi-Yau threefold $X$. It counts the number of stable maps of connected domain curves to $X$ in any given non-zero two-homology class; because of the existence of automorphisms, one has to perform a weighted count by dividing by the orders of the automorphism groups, and hence GromovWitten invariants are in general rational numbers. The Gopakumar-Vafa conjecture asserts particular integrality properties of $\mathcal{F}_{X}^{\text {top }}(\tau, Q)$, and in particular the existence of integers $n_{\left(q_{a}\right)}^{g}=n_{\left(q_{a}\right)}^{g}(X)$, which enumerate BPS states of wrapped membranes in $X$ such that

$$
\mathcal{F}_{X}^{\mathrm{top}}(\tau, Q)=\sum_{\left(q_{a}\right) \in \Gamma^{*}} \sum_{g=0}^{\infty} \sum_{k=1}^{\infty} \frac{1}{k} n_{\left(q_{a}\right)}^{g}(2 \sin (\pi k \tau))^{2 g-2} \mathrm{e}^{-k q_{a} \phi^{a}},
$$

where for a given curve class $\mathcal{C}$ labelled by $\left(q_{a}\right) \in \Gamma^{*}=H_{2}(X, \mathbb{Z}) \backslash\{0\}$ there are only finitely many non-zero $n_{\left(q_{a}\right)}^{g} \in \mathbb{Z}$ in genera $g \geq 0$. Here $\phi^{a}:=\int_{\mathcal{C}^{a}} \omega$ are the Kähler parameters of $X$. Let us regard $q$ as an element of $S U(2)$ represented by the diagonal matrix $\operatorname{diag}\left(q, q^{-1}\right)$; then the sine functions in (3.21) can be interpreted as characters of $S U(2)$ representations and we define integers $N_{\left(q_{a}\right)}^{g}$ such that

$$
\sum_{g=0}^{\infty}(-1)^{g} n_{\left(q_{a}\right)}^{g}(2 \sin (\pi \tau))^{2 g}=\sum_{g=0}^{\infty} N_{\left(q_{a}\right)}^{g} \sum_{i=0}^{g} q^{g-2 i},
$$


which is understood as a change of integral basis in the representation ring of $S U(2)$. Analogously to [43], we then have

$$
\begin{aligned}
\mathcal{F}_{X}^{\mathrm{top}}(\tau, Q) & =\sum_{\left(q_{a}\right) \in \Gamma^{*}} \sum_{g=0}^{\infty} \sum_{k=1}^{\infty} \frac{(-1)^{g-1} N_{\left(q_{a}\right)}^{g}}{k\left(q^{k / 2}-q^{-k / 2}\right)^{2}} \mathrm{e}^{-k q_{a} \phi^{a}} \sum_{i=0}^{g} q^{k(g-2 i)} \\
& =\sum_{\left(q_{a}\right) \in \Gamma^{*}} \sum_{g=0}^{\infty} \sum_{k=1}^{\infty} \frac{(-1)^{g-1} N_{\left(q_{a}\right)}^{g}}{k} \mathrm{e}^{-k q_{a} \phi^{a}} \sum_{n=0}^{\infty}(n+1) q^{k n} \sum_{i=0}^{g} q^{k(g-2 i+1)} \\
& =\sum_{\left(q_{a}\right) \in \Gamma^{*}} \sum_{g=0}^{\infty}(-1)^{g} N_{\left(q_{a}\right)}^{g} \sum_{i=0}^{g} \sum_{n=0}^{\infty}(n+1) \log \left(1-q^{g-2 i+n+1} \mathrm{e}^{-q_{a} \phi^{a}}\right) .(3.22)
\end{aligned}
$$

In calculating the last line in Eq. (3.22) we can use the Ruelle function $\mathcal{R}(s)$ from Appendix $\mathrm{A}$ and the identity

$$
\sum_{n=1}^{\infty} n \log \left(1-q^{n+\varepsilon}\right)=\sum_{n=1}^{\infty} \log \mathcal{R}(s=(n+\varepsilon)(1-\mathrm{i} \varrho(\tau))),
$$

for $\varepsilon \in \mathbb{C}$. The generating series of disconnected Gromov-Witten invariants is given by the topological string partition function $\mathcal{Z}_{X}^{\text {top }}(\tau, \phi)=\exp \left(-\mathcal{F}_{X}^{\text {top }}(\tau, Q)\right)$. Setting $j=g / 2$ and $N_{\left(q_{a}\right), j}:=N_{\left(q_{a}\right)}^{g}$, the Gopakumar-Vafa conjecture can be reformulated as the infinite product formula (see also [33])

$$
\begin{aligned}
\mathcal{Z}_{X}^{\text {top }}(\tau, \phi) & =\prod_{\left(q_{a}\right) \in \Gamma^{*}, n>0} \prod_{\substack{2 j \geq 0 \\
-j \leq m \leq j}}\left[\mathcal{R}\left(s=\left(n+2 m+q_{a} \widehat{\phi}^{a}\right)(1-\mathrm{i} \varrho(\tau))\right)\right]^{(-1)^{2 j+1} N_{\left(q_{a}\right), j}} \\
& =\prod_{\left(q_{a}\right) \in \Gamma^{*}, n>0} \prod_{\substack{2 j \geq 0 \\
-j \leq m \leq j}}\left(1-q^{n+2 m} \mathrm{e}^{-q_{a} \phi^{a}}\right)^{(-1)^{2 j+1} n N_{\left(q_{a}\right), j}}
\end{aligned}
$$

This formula reveals the geometric meaning of the membrane contributions to the black hole partition function (3.19).

Resummation of the $(\mathbf{4 , 0 )}$ elliptic genus. We can turn this last calculation around to rewrite the expression (3.19) for the black hole partition function in the form of spectral functions, and rearrange it into a more geometric expansion in terms of BPS invariants rather than the multiplicities $N_{\left(q_{a}\right), j_{L}, j_{R}}$ of irreducible representations. Dropping the ground state, neutral supergravity mode, and anti-membrane contributions to the free energy $\mathcal{F}_{X}(\tau, \phi)=$ 
$-\log \mathcal{Z}_{X}(\tau, \phi)$ in Eq. (3.19), we have

$$
\begin{aligned}
& \mathcal{F}_{X}(\tau, \phi)=\sum_{\substack{\left(q_{a}\right) \in \Gamma^{*} \\
p^{a}, n>0}} \sum_{\substack{2 j_{L, R} \geq 0 \\
-j_{L} \leq m_{L} \leq j_{L}}}(-1)^{2 j_{L}+2 j_{R}}\left(2 j_{R}+1\right) N_{\left(q_{a}\right), j_{L}, j_{R}} \\
& \times n \log \left(1-q^{n+\frac{1}{2} q_{a} p^{a}+2 m_{L}} \mathrm{e}^{-q_{a} \phi^{a}}\right) \\
& =-\sum_{\substack{\left(q_{a}\right) \in \Gamma^{*} \\
p^{a}, n, k>0}} \sum_{\substack{2 j_{L}, R \geq 0 \\
-j_{L} \leq m_{L} \leq j_{L}}}(-1)^{2 j_{L}+2 j_{R}}\left(2 j_{R}+1\right) N_{\left(q_{a}\right), j_{L}, j_{R}} \\
& \times \frac{n}{k} q^{k\left(n+\frac{1}{2} q_{a} p^{a}+2 m_{L}\right)} \mathrm{e}^{-k q_{a} \phi^{a}} \\
& =-\sum_{\substack{\left(q_{a}\right) \in \Gamma^{*} \\
p^{a}, n, k>0}} \sum_{\substack{2 j_{L, R} \geq 0 \\
-j_{L} \leq m_{L} \leq j_{L}}} \frac{(-1)^{2 j_{L}+2 j_{R}}\left(2 j_{R}+1\right) N_{\left(q_{a}\right), j_{L}, j_{R}} n}{k\left(1-q^{k\left(\frac{1}{2} q_{a} p^{a}+2 m_{L}\right)} \mathrm{e}^{\left.-k q_{a} \phi^{a}\right)}\right.} \\
& \times\left(q^{k\left(n+\frac{1}{2} q_{a} p^{a}+2 m_{L}\right)} \mathrm{e}^{-k q_{a} \phi^{a}}-q^{k\left(n+q_{a} p^{a}+4 m_{L}\right)} \mathrm{e}^{-2 k q_{a} \phi^{a}}\right) \\
& =\sum_{\substack{\left(q_{a}\right) \in \Gamma^{*} \\
p^{a}, n>0}} \sum_{\substack{2 j_{L, R} \geq 0 \\
-j_{L} \leq m_{L} \leq j_{L}}}(-1)^{2 j_{L}+2 j_{R}}\left(2 j_{R}+1\right) N_{\left(q_{a}\right), j_{L}, j_{R}} \\
& \times n \log \left[\frac{\prod_{l=n}^{\infty}\left(1-q^{l+\frac{1}{2} q_{a} p^{a}+2 m_{L}} \mathrm{e}^{-q_{a} \phi^{a}}\right)}{\prod_{l=n}^{\infty}\left(1-q^{l+1+\frac{1}{2} q_{a} p^{a}+2 m_{L}} \mathrm{e}^{-q_{a} \phi^{a}}\right)}\right] \\
& =\sum_{\left(q_{a}\right) \in \Gamma^{*}, p^{a}>0} \sum_{\substack{2 j_{L, R} \geq 0 \\
-j_{L} \leq m_{L} \leq j_{L}}}(-1)^{2 j_{L}+2 j_{R}}\left(2 j_{R}+1\right) N_{\left(q_{a}\right), j_{L}, j_{R}} \\
& \times \log \prod_{n=1}^{\infty}\left(1-q^{n+\frac{1}{2} q_{a} p^{a}+2 m_{L}} \mathrm{e}^{-q_{a} \phi^{a}}\right)^{n} \\
& =\sum_{\left(q_{a}\right) \in \Gamma^{*}, p^{a}>0} \sum_{\substack{2 j_{L, R} \geq 0 \\
-j_{L} \leq m_{L} \leq j_{L}}}(-1)^{2 j_{L}+2 j_{R}}\left(2 j_{R}+1\right) N_{\left(q_{a}\right), j_{L}, j_{R}} \\
& \times \log \prod_{n=1}^{\infty} \mathcal{R}\left(s=\left(n+q_{a}\left(\frac{p^{a}}{2}-\widehat{\phi}^{a}\right)+2 m_{L}\right)(1-\mathrm{i} \varrho(\tau))\right) .
\end{aligned}
$$

The Gopakumar-Vafa invariants $n_{r}\left(\left(q_{a}\right)\right) \in \mathbb{Z}$ which count M2-branes wrapping genus $r$ curves with charges $q_{a}$ are defined in terms of the quantum degeneracies $N_{\left(q_{a}\right), j_{L}, j_{R}}$ via [28, 26]

$$
\sum_{r=0}^{\infty}(-1)^{r} n_{r}\left(\left(q_{a}\right)\right)(2 \sin (\pi \tau))^{2 r}=\sum_{\substack{2 j_{L, R} \geq 0 \\-j_{L} \leq m_{L} \leq j_{L}}}(-1)^{2 j_{L}+2 j_{R}}\left(2 j_{R}+1\right) N_{\left(q_{a}\right), j_{L}, j_{R}} q^{2 m_{L}}
$$

Hence BPS invariants of $X$ can be obtained by keeping only the contributions from states 
with $j_{L}=0$, and comparing Eqs. (3.24) and (3.25) we obtain

$$
\begin{aligned}
\mathcal{F}_{X}^{0}(\tau, \phi) & =\sin (2 \pi \tau) \sum_{\left(q_{a}\right) \in \Gamma^{*}, p^{a}>0} \sum_{r=1}^{\infty}(-1)^{r} n_{r}\left(\left(q_{a}\right)\right)(2 \sin (\pi \tau))^{2 r} \\
& \times \sum_{n=1}^{\infty} \log \mathcal{R}\left(s=\left(n+q_{a}\left(\frac{p^{a}}{2}-\widehat{\phi}^{a}\right)\right)(1-\mathrm{i} \varrho(\tau))\right) .
\end{aligned}
$$

\section{D-brane partition functions and (2,2) elliptic genera}

\subsection{Black hole partition functions in the Type IIA frame}

We shall now explain how to compute the contributions to the microscopic black hole entropy in the setting of Sect. 3 by enumerating BPS bound states of D-branes inside the compact Calabi-Yau threefold $X$. The same techniques are then employed for the computation of the supergravity elliptic genus in the generalization of these considerations to BPS black holes in $\mathcal{N}=2$ compactifications on more general Calabi-Yau manifolds $X$. One particularly noteworthy feature of this formalism will be the natural appearance of integrable highest weight representations of affine Lie algebras on the homology of the D-brane moduli spaces, as anticipated from the $A d S_{3} / C F T_{2}$ correspondence. The characters of these modules compute the holomorphic parts of the torus partition functions in the corresponding two-dimensional superconformal field theory and are identified with black hole partition functions as before. Since the M5-brane wraps the circle $S_{m}^{1}$ and the M-momentum is along $S_{m}^{1}$, we can take $S_{m}^{1}$ to be the M-circle and regard the M5-M2 system from Sect. 3 as an M-theory lift of a system of bound D4-D2-D0 states wrapping the divisor $M$ in Type IIA string theory compactified on the Calabi-Yau manifold $X$. These supersymmetric ground states can be regarded as excitations in the worldvolume theory on the D4-brane. Below we will demonstrate how they may be regarded as multiparticle chiral primary states in $A d S_{3}$. In the large volume limit, this theory is the $U(1)$ Vafa-Witten topologically twisted $\mathcal{N}=4$ gauge theory [52] on $M$. D0-branes are then identified as instantons in this gauge theory which correspond to massless supergravity modes, where the Kaluza-Klein momentum on $S_{m}^{1}$ is the instanton number

$$
q_{0}=n=\frac{1}{8 \pi^{2}} \int_{M} F \wedge F
$$

while the $U(1)$ fluxes on the D4-brane carried by D2-branes correspond to M2-branes wrapping holomorphic curves on its worldvolume in $X$ so that the membrane charges in the M-theory lift are magnetic monopole numbers

$$
q_{a}=C_{a b} u^{b}=\frac{1}{2 \pi} \int_{M} F \wedge \Sigma_{a}
$$

By self-duality one has $q_{a}=0$ for all $a>b_{2}^{+}(M)$. In this setting, the Hilbert space of BPS states $\mathcal{H}_{q, p}(X)$ is the cohomology ring of the moduli space $\mathcal{M}_{n, u}(M)$ of $U(1)$ instantons on 
$M$ with second Chern class $n$ and first Chern class $u$, and the degeneracy of bound states

$$
d(q, p)=\chi\left(\mathcal{M}_{n, u}(M)\right)
$$

equals the Euler character of the instanton moduli space. The black hole partition function (3.5) is then the generating function for these Euler numbers given by

$$
\mathcal{Z}_{M}(\tau, z)=\sum_{n=0}^{\infty} \sum_{u \in \Gamma} \chi\left(\mathcal{M}_{n, u}(M)\right) q^{n} \prod_{a=1}^{b_{2}^{+}(M)} w_{a}^{u^{a}},
$$

where as before we set $q=\mathrm{e}^{-\phi_{0}}=\mathrm{e}^{2 \pi \mathrm{i} \tau}$ and we define $w_{a}=\mathrm{e}^{-C_{a b} \phi^{b}}=: \mathrm{e}^{2 \pi \mathrm{i} z_{a}}$. The smooth Gieseker compactification of the instanton moduli space 7 admits a factorization [17]

$$
\mathcal{M}_{n, u}(M)=M^{\left[n-n_{u}\right]} \times \operatorname{Pic}_{n_{u}}(M),
$$

where the punctual Hilbert scheme $M^{\left[n-n_{u}\right]}$ from Sect. 2.3 parametrizing D0-branes on $M$ is a smooth complex manifold of dimension $2\left(n-n_{u}\right)$, and $\operatorname{Pic}_{n_{u}}(M)$ is the Picard lattice which parametrizes D2-branes corresponding to holomorphic line bundles on $M$ of first Chern class $u$. The charge $n_{u}=\frac{1}{2} u^{a} C_{a b} u^{b}+\frac{1}{2} u^{a} c_{1}(M)_{a}$ is the curvature induced D0-brane charge on the D4-brane. The sum over instanton numbers can be performed explicitly by using Göttsche's formula from Sect. 2.3, and in this way the black hole partition function (4.1) is computed to be [22, 30, 17]

$$
\mathcal{Z}_{M}(\tau, z)=\frac{\Theta_{\Gamma}(\tau, z)}{\hat{\eta}(\tau)^{\chi(M)}}=\Theta_{\Gamma}(\tau, z) \mathcal{R}(s=1-\mathrm{i} \varrho(\tau))^{-\chi(M)},
$$

where

$$
\Theta_{\Gamma}(\tau, z)=\sum_{u \in \Gamma} q^{\frac{1}{2} u^{a} C_{a b} u^{b}} \prod_{a=1}^{b_{2}^{+}(M)} w_{a}^{u^{a}}
$$

is a Riemann theta-function on the magnetic M2/D2-brane charge lattice $\Gamma=H^{2}(M, \mathbb{Z})$.

The refined black hole partition function (3.9) can be computed in the Type IIA frame by considering the gauge theory on the full five-dimensional D4-brane worldvolume $M \times S_{m}^{1}$. In this case, instantons on the divisor $M$ become solitons on $M \times S_{m}^{1}$ whose worldlines wrap around the circle $S_{m}^{1}$. Then the refinement parameter is identified as $y=\mathrm{e}^{-\beta}=: \mathrm{e}^{2 \pi \mathrm{i} \rho}$, where $\beta$ is the radius of the Euclidean temporal circle $S_{m}^{1}$ that is also used to associate a Hilbert space $\mathcal{H}_{q, p}(X)$ with $M \times S_{m}^{1}$, which is additionally graded by angular momentum and R-charge. The refined black hole partition function is now computed from an index in a supersymmetric quantum mechanics on the instanton moduli space $\mathcal{M}_{n, u}(M)$, which includes a product over all Kaluza-Klein modes of the fluctuations around the D4-D2-D0 bound states. In this case the protected spin character

$$
d_{y}(q, p)=\chi_{y}\left(\mathcal{M}_{n, u}(M)\right)
$$

\footnotetext{
${ }^{7}$ Here we use the standard identification of D-branes with (torsion free) sheaves on large radius Calabi-Yau manifolds.
} 
equals the Hirzebruch $\chi_{y}$-genus of the instanton moduli space [1]. Recall from Sect. 2.1 that it is given by a sum of the form (2.8) over components of the BPS Hilbert space, where the degrees $(j, k)$ are related to the spin and R-charge by $\left(J_{3}, J_{0}\right)=\frac{1}{2}(j+k, j-k)$. In particular, for $y=0$ it computes the geometric genus

$$
\chi_{0}\left(\mathcal{M}_{n, u}(M)\right)=\chi\left(\mathcal{M}_{n, u}(M), \mathcal{O}_{\mathcal{M}}\right)
$$

of $\mathcal{M}=\mathcal{M}_{n, u}(M)$, which gives the degeneracy of BPS states with equal spin and R-charge, while for $y=1$ we recover the Witten index $\chi_{1}\left(\mathcal{M}_{n, u}(M)\right)=\chi\left(\mathcal{M}_{n, u}(M)\right)$. It can be computed in general in terms of characteristic classes via Eq. (2.10) with $X=\mathcal{M}_{n, u}(M)$ and $d=2\left(n-n_{u}\right)$. The refined black hole partition function (3.9) is then given by the generating function

$$
\mathcal{Z}_{M}(\tau, z, \rho)=\sum_{n=0}^{\infty} \sum_{u \in \Gamma} \chi_{y}\left(\mathcal{M}_{n, u}(M)\right) q^{n} \prod_{a=1}^{b_{2}^{+}(M)} w_{a}^{u^{a}}
$$

From the perspective of the five-dimensional supersymmetric gauge theory on the D4-brane, the counting parameter $q=\beta^{2} \Lambda^{2}$ is determined by the dynamical scale $\Lambda$ of the fourdimensional gauge theory which arises in the dimensional reduction $\beta=0$. The sum over instanton numbers can again be performed explicitly by using results from Sect. 2.3 and Eq. (2.20) to get

$$
\begin{aligned}
\mathcal{Z}_{M}(\tau, z, \rho) & =\Theta_{\Gamma}(\tau, z) \prod_{j=0}^{2} \prod_{n=1}^{\infty}\left(1-y^{j+n} q^{n}\right)^{(-1)^{j+1} \chi^{j}(M)} \\
& =\Theta_{\Gamma}(\tau, z) \prod_{j=0}^{2} \mathcal{R}(\widehat{s}=2 \pi \mathrm{i}(\tau+j \rho)(1-\mathrm{i} \varrho(\tau))-2 \pi \mathrm{i} \rho)^{(-1)^{j+1} \chi^{j}(M)} .
\end{aligned}
$$

\subsection{Black hole components of chiral primary states}

We can alternatively consider the D4-D2-D0 states above from the perspective of the D0branes. This point of view can be used to study the black hole components of chiral primary fields of an $\mathcal{N}=2$ superconformal field theory in string theory compactified on the CalabiYau threefold $X$; recall that chiral primary fields form an algebra [42, 54]. D0-branes in the $A d S_{2} \times S^{2} \times X$ attractor geometry of an extremal Calabi-Yau black hole with D4-brane charges $p^{a}$ are described by a superconformal quantum mechanics [25]. This superconformal theory contains a large degeneracy of chiral primary bound states. The degeneracy arises from D0-branes in the lowest Landau level which tile the $S^{2} \times X$ horizon, and such a multiD0-brane conformal field theory $C F T_{1}$ is holographically dual to Type IIA string theory on $A d S_{2} \times S^{2} \times X$. Of course, from the perspective of $A d S_{2} / C F T_{1}$ holography there is no a priori reason why the black hole degeneracy should exhibit any form of modularity. On the other hand, modular properties are anticipated by the (conjectural) S-duality of the D4-brane gauge theory [52]. Moreover, one expects that BPS states of D-branes on Calabi-Yau manifolds have an algebraic structure akin to generalised Kac-Moody algebras, 
generalising the geometric construction of highest modules of affine Lie algebras that we discussed in Sect. 2.3. Our rewriting below of the D-brane quantum partition functions in terms of spectral Ruelle functions makes this modularity and algebraic structure manifest.

We consider the case when a D2-brane wraps the horizon $S^{2}$ and carries $N$ units of magnetic flux, corresponding to $N$ units of D0-brane charge. The D2-brane can be considered as a point particle in $X$ with a two-form magnetic field $c_{1}([M])=p^{a} \Sigma_{a}$. The magnetic field divides the Calabi-Yau threefold $X$ into $D$ cells corresponding to the lowest Landau levels; in this setting the natural counting function is the elliptic genus determined by Eq. (2.11) with

$$
\begin{aligned}
\chi(M ; q, y) & =y^{-1} \int_{X}\left[\frac{\mathcal{R}\left(\widehat{s}=-c_{1}([M])(1-\mathrm{i} \varrho(\tau))\right)}{\mathcal{R}\left(\widehat{s}=\left(2 \pi \mathrm{i} z-c_{1}([M])\right)(1-\mathrm{i} \varrho(\tau))\right)}\right] \\
& \times\left[\frac{\mathcal{R}\left(\widehat{s}=\left(2 \pi \mathrm{i} \tau+c_{1}([M])\right)(1-\mathrm{i} \varrho(\tau))\right)}{\mathcal{R}\left(\widehat{s}=\left(2 \pi \mathrm{i}(\tau-z)+c_{1}([M])\right)(1-\mathrm{i} \varrho(\tau))\right)}\right] \\
& \times \prod_{j=1}^{3} x_{j}\left[\frac{\mathcal{R}\left(\widehat{s}=\left(2 \pi \mathrm{i} z-x_{j}\right)(1-\mathrm{i} \varrho(\tau))\right)}{\mathcal{R}\left(\widehat{s}=-x_{j}(1-\mathrm{i} \varrho(\tau))\right)}\right] \\
& \times\left[\frac{\mathcal{R}\left(\widehat{s}=\left(2 \pi \mathrm{i}(\tau-z)+x_{j}\right)(1-\mathrm{i} \varrho(\tau))\right)}{\mathcal{R}\left(\widehat{s}=\left(2 \pi \mathrm{i} \tau+x_{j}\right)(1-\mathrm{i} \varrho(\tau))\right)}\right] .
\end{aligned}
$$

From our discussion of the modularity properties of the field theory elliptic genus from Sect. 2.1, this exhibits the D-brane partition functions as weak Jacobi forms. However, here we shall instead proceed in a more direct way.

The chiral primary conditions can be written as $\nabla \omega=\nabla^{*} \omega=0$, where $\omega$ is a $(j, k)$-form on $X$, and $\nabla$ is a holomorphic covariant derivative with connection that generates the magnetic field. Solutions of this equation are in one-to-one correspondence with the elements of $H^{k}\left(X, \Omega_{X}^{j} \otimes[M]\right)$. By the Serre vanishing theorem, the cohomology group $H^{k}\left(X, \Omega_{X}^{j} \otimes[M]\right)$ vanishes for $k>0$ and sufficiently large magnetic field $c_{1}([M])$.

The upshot is that we need compute the cohomology $H^{k}\left(X, \Omega_{X}^{j} \otimes[M]\right)$ for the moduli space of a D2-brane on $X$, and the black hole entropy counting can be reduced to a cohomology problem as before. In some cases one can compute the dimension of $H^{k}\left(X, \Omega_{X}^{j} \otimes[M]\right)$ using mirror symmetry 8 while

$$
b_{j}:=\operatorname{dim} H^{0}\left(X, \Omega_{X}^{j} \otimes[M]\right)
$$

\footnotetext{
${ }^{8}$ If $X$ is mirror to $Y$, then by homological mirror symmetry a D0-brane in $X$ is mirror to a three-torus $T^{3}$ in $Y$. Moreover, the moduli space of a D3-brane wrapping such a $T^{3}$ in $Y$ is $X$. One can thus compute $H^{k}\left(X, \Omega_{X}^{j} \otimes[M]\right)$ and therefore the black hole entropy using homological mirror symmetry.
} 
can be obtained by using the Riemann-Roch formula to get (see also [45, 25])

$$
\begin{aligned}
b_{0} & =\int_{X}\left(\frac{c_{1}([M]) \wedge c_{1}([M]) \wedge c_{1}([M])}{6}+\frac{c_{2}(X) \wedge c_{1}([M])}{12}\right)=D+\frac{1}{12}\left(c_{2}\right)_{a} p^{a}=b_{3}, \\
b_{1} & =\int_{X}\left(\frac{c_{1}([M]) \wedge c_{1}([M]) \wedge c_{1}([M])}{2}-\frac{3 c_{2}(X) \wedge c_{1}([M])}{4}+\frac{c_{3}(X)}{2}\right) \\
& =3 D-\frac{3}{4}\left(c_{2}\right)_{a} p^{a}-\frac{1}{2} \chi(X), \\
b_{2} & =\int_{X}\left(\frac{c_{1}([M]) \wedge c_{1}([M]) \wedge c_{1}([M])}{2}-\frac{3 c_{2}(X) \wedge c_{1}([M])}{4}-\frac{c_{3}(X)}{2}\right) \\
& =3 D-\frac{3}{4}\left(c_{2}\right)_{a} p^{a}+\frac{1}{2} \chi(X) .
\end{aligned}
$$

We now have to count the multiparticle primaries, by first choosing a basis of states. As in Sect. 2.2, we can partition the D0-branes into $k$ clusters, with the $l$-th cluster containing $n_{l}$ D0-branes where $\sum_{l=1}^{k} n_{l}=N$. Each cluster forms a wrapped D2-brane with $n_{l}$ units of magnetic flux (we ignore the possibility of multiply wrapped D2-brane bound states). Then each of the $k$ wrapped D2-branes can sit in one of the $b_{0}+b_{1}+b_{2}+b_{3}$ chiral primary states. The counting of configurations is in fact in one-to-one correspondence with the counting of states for a conformal field theory with $b_{0}+b_{2}$ bosons and $b_{1}+b_{3}$ fermions of total momentum $n=n_{l}$. The partition function of the appropriate conformal field theory can be calculated by taking the trace over chiral primary states, with the result

$$
\mathcal{Z}_{\mathrm{CFT}}(\tau)=\prod_{n=1}^{\infty} \frac{\left(1+q^{n}\right)^{b_{1}+b_{3}}}{\left(1-q^{n}\right)^{b_{0}+b_{2}}}=\frac{[\mathcal{R}(s=1-\mathrm{i} \varrho(\tau)+\mathrm{i} /(2 \operatorname{Im} \tau))]^{b_{1}+b_{3}}}{[\mathcal{R}(s=1-\mathrm{i} \varrho(\tau))]^{b_{0}+b_{2}}}
$$

\subsection{Microscopic black hole entropy}

We will now show that (4.3) is in perfect agreement with the microscopic M5-brane computation of the black hole entropy in the two-dimensional $(4,0)$ superconformal field theory derived by [45]; it also agrees with the macroscopic supergravity result [22]. The macroscopic entropy of these black holes should coincide with the asymptotic growth of the degeneracy by the Boltzmann relation

$$
S(q, p)=\log d(q, p)
$$

for $q, p \gg 1$. On the other hand, the asymptotic growth of the microscopic degeneracy evaluated via the Cardy formula gives the black hole entropy

$$
S(q, p)=2 \pi \sqrt{\frac{1}{6} c_{L} \tilde{q}_{0}},
$$

where $\tilde{q}_{0}$ is the momentum remaining of the total M-momentum $q_{0}$ to freely distribute in the M-theory frame; one can in fact improve the Cardy formula (4.13) by including a degeneracy prefactor [15]. In the present case one finds [22]

$$
S(q, p)=2 \pi \sqrt{\left(D+\frac{1}{6}\left(c_{2}\right)_{a} p^{a}\right)\left(q_{0}+\frac{1}{12} q_{a} D^{a b} q_{b}\right)}
$$


for $q, p \gg 1$, where the first term under the square root is $\frac{1}{6} c_{L}=\frac{1}{6} \chi(M)$ while the second term is the number $\tilde{q}_{0}$ of D0-branes coming from the expansion of $\hat{\eta}(\tau)^{-\chi(M)}$ which gives the contributions from massless neutral supergravity modes.

\subsection{Representations of Heisenberg algebras}

The expression (4.3) is also the partition function of a chiral two-dimensional conformal field theory of $N=\chi(M)$ free bosons with $N_{+}=b_{2}^{+}(M)$ degrees of freedom on the charge lattice $\Gamma=H^{2}(M, \mathbb{Z})$. We elucidate this observation by calculating the black hole partition function from a sigma-model based on a generalized chiral algebra [9]. Consider the Heisenberg Lie algebra $\widehat{\mathfrak{h}}(\Gamma)$ based on the lattice $\Gamma$ with the intersection form $C_{a b}$. It has generators $\alpha_{n}^{a}$, $n \in \mathbb{Z}, a=1, \ldots, N_{+}$which satisfy the commutation relations $\left[\alpha_{n}^{a}, \alpha_{m}^{b}\right]=\delta_{n+m, 0} C^{a b}$.

Let $\mathcal{F}(\Gamma)$ be the irreducible Fock space representation of $\widehat{\mathfrak{h}}(\Gamma)$; by Sect. 2.3 it can be identified geometrically with the cohomology ring of the instanton moduli space $\bigoplus_{n, u} H^{\bullet}\left(\mathcal{M}_{n, u}(M), \mathbb{C}\right)$. Define a sigma-model whose target space is the degree two $L^{2}$-cohomology of $M$ modulo integral elements, so that the momentum lattice is $\Gamma$, and whose Hamiltonian is given by

$$
L_{0}=\frac{1}{2} \alpha_{0}^{a} C_{a b} \alpha_{0}^{b}+\sum_{n=1}^{\infty} \alpha_{-n}^{a} C_{a b} \alpha_{n}^{b} .
$$

Then the black hole partition function (4.3) can be reproduced from the one-loop partition function of the sigma-model

$$
\chi_{M}(\tau, z)=\operatorname{Tr}_{\mathcal{F}(\Gamma)} q^{L_{0}} \mathrm{e}^{-\zeta_{a} J_{0}^{a}}=\hat{\eta}(\tau) \mathcal{Z}_{M}(\tau, z),
$$

where $\zeta_{a}=C_{a b} \phi^{b}=-2 \pi \mathrm{i} z_{a}$ and $J_{0}^{a}=\alpha_{0}^{a}$. In the M-theory frame, the extra free boson partition function $\hat{\eta}(\tau)$ arises from the remaining part of the reduced self-dual three-form field. The generalized chiral algebra is thus associated with the sum of Heisenberg algebras $\widehat{\mathfrak{h}} \oplus \widehat{\mathfrak{h}}(\Gamma)$.

\subsection{Toric Calabi-Yau black holes}

The D-brane partition function (4.3) is derived in [22, 30, 17] for a large class of noncompact toric manifolds $M$, regarded as divisors in the total space of the canonical line bundle $X=K_{M}$. The non-compact D-brane worldvolumes in question are the HirzebruchJung spaces, which are toric minimal resolutions of $(k, l)$ quotient singularities $\mathbb{C}^{2} / \mathfrak{C}_{k, l}$ with the cyclic group $\mathfrak{C}_{k, l} \cong \mathbb{Z}_{k}$ parametrized by a pair of coprime integers $(k, l)$ with $k>l>0$. The intersection matrix $C_{a b}$ in this case is parametrized by a finite sequence $\left\{e_{i}\right\}_{i=1}^{\ell}$ of integers $e_{i} \geq 2$, which appear in the continued fraction expansion

$$
\frac{k}{l}=\left[e_{1}, \ldots, e_{\ell}\right]:=e_{1}-\frac{1}{e_{2}-\frac{1}{e_{3}-\frac{1}{\ddots \cdot e_{\ell-1}-\frac{1}{e_{\ell}}}}}
$$


as

$$
C=\left(C_{a b}\right)=\left(\begin{array}{ccccc}
e_{1} & -1 & 0 & \cdots & 0 \\
-1 & e_{2} & -1 & \cdots & 0 \\
0 & -1 & e_{3} & \cdots & 0 \\
\vdots & \vdots & \vdots & \ddots & \vdots \\
0 & 0 & 0 & \cdots & e_{\ell}
\end{array}\right)
$$

We will refer to $C$ as a generalized Cartan matrix. The topological data of $M$ are then $\chi(M)=\ell+1, b_{2}^{+}(M)=\ell$ and $\Gamma=H^{2}(M, \mathbb{Z})=\mathbb{Z}^{\ell}$. Let us construct two sequences of integers $\left\{k_{i}\right\}_{i=0}^{\ell}$ and $\left\{l_{i}\right\}_{i=0}^{\ell}$ defined by the continued fraction expansions

$$
\frac{k_{i}}{l_{i}}=\left[e_{1}, \ldots, e_{i-1}\right]
$$

for $i=2, \ldots, \ell+1$, with the initial values

$$
k_{0}=0, k_{1}=1 \quad \text { and } \quad l_{0}=-1, l_{1}=0 .
$$

In particular, $k_{\ell}=k$ and $l_{\ell}=l$. They satisfy the recursion relations

$$
k_{i+1}=e_{i} k_{i}-k_{i-1} \quad \text { and } \quad l_{i+1}=e_{i} l_{i}-l_{i-1}
$$

for $i=1, \ldots, \ell$.

Let $\left(\varepsilon_{1}, \varepsilon_{2}\right)$ be infinitesimal parameters of the abelian subgroup $T=U(1) \times U(1)$ of the Lorentz group $S O(4) \cong S U(2) \times S U(2)$ of $\mathbb{R}^{4} \cong \mathbb{C}^{2}$. The action of this torus on the singular variety $\mathbb{C}^{2} / \mathfrak{C}_{k, l}$ lifts to a torus action on the resolved variety whose weights $\left(\varepsilon_{1}^{(i)}, \varepsilon_{2}^{(i)}\right)$ on each of the $\ell+1$ torus-invariant patches $U_{i} \cong \mathbb{C}^{2}$ of $M$ are given by [9]

$$
\varepsilon_{1}^{(i)}=-\left(k l_{i}-l k_{i}\right) \varepsilon_{1}-k_{i} \varepsilon_{2} \quad \text { and } \quad \varepsilon_{2}^{(i)}=\left(k l_{i+1}-l k_{i+1}\right) \varepsilon_{1}+k_{i+1} \varepsilon_{2}
$$

for $i=0,1, \ldots, \ell$. The $T$-action further lifts to a torus action on the D-brane moduli spaces (4.2). In the following we will mostly work in the antidiagonal limit $\varepsilon_{1}=-\varepsilon_{2}=$ : $\hbar$ of this toric action; in this case the holomorphic two-form of $\mathbb{C}^{2}$ is preserved by the $T$-action.

The black hole partition function (4.3) in this instance now follows from a localization calculation of the equivariant Euler character $\chi_{T}\left(\mathcal{M}_{n, u}(M)\right)$ of the moduli space of BPS configurations in the sector labelled by $(n, u)$; equivariant localization is used here to properly define characters of the non-compact instanton moduli space (4.2), and it is understood as a calculation in the $\Omega$-deformation of the underlying supersymmetric gauge theory wherein the gauge symmetry is coupled to the abelian isometries generated by $T$. Localization on the D-brane moduli space (4.2) computes the partition function (4.1) as a sum over the isolated $T$-fixed points of the Hilbert scheme $M^{[n]}$, which decompose into contributions from each toric open set $U_{i} \cong \mathbb{C}^{2}$. Thus the equivariant version of (4.1) can be computed from the blow-up formula [27, Cor. 5.12]

$$
\mathcal{Z}_{M}\left(\varepsilon_{1}, \varepsilon_{2} ; \tau, z\right)=\sum_{u \in \mathbb{Z}^{\ell}} q^{\frac{1}{2} u^{a} C_{a b} u^{b}} \prod_{a=1}^{\ell} w_{a}^{u^{a}} \prod_{i=0}^{\ell} \mathcal{Z}_{\mathbb{C}^{2}}\left(\varepsilon_{1}^{(i)}, \varepsilon_{2}^{(i)} ; \tau\right)
$$


The $T$-fixed points of $M^{[n]}$ over $U_{i} \cong \mathbb{C}^{2}$ are parametrized by Young diagrams $Y$ with $|Y|=n$ boxes [46]. The classes which enter in the denominator of the localization formula in this case cancel against the equivariant Euler characteristic classes at each fixed point. Hence the contributions from each patch $U_{i}$ are independent of the equivariant parameters and coincide with the generating function for Young diagrams given by Euler's formula

$$
\mathcal{Z}_{\mathbb{C}^{2}}\left(\varepsilon_{1}, \varepsilon_{2} ; \tau\right)=\sum_{Y} q^{|Y|}=\hat{\eta}(\tau)^{-1}
$$

independently of $\left(\varepsilon_{1}, \varepsilon_{2}\right)$, and the result (4.3) follows.

In the following we will extend this reasoning to the computation of quantum black hole degeneracies which are given by Fourier coefficients of elliptic genera of the D-brane moduli spaces. Working in an equivariant setting has the advantage that the computation of the equivariant elliptic genera for symmetric products, and hence for the D-brane moduli spaces, can be naturally reduced to infinite product expansions which may be compared with those from Sect. 3. Let us illustrate this procedure explicitly for the equivariant version of the refined black hole partition function (4.8) which is derived by applying the localization theorem in $T$-equivariant cohomology to the integral (2.10) over the D-brane moduli space $X=\mathcal{M}_{n, u}(M)$; it generally depends on the equivariant parameters $\left(\varepsilon_{1}, \varepsilon_{2}\right)$ and is given by suitable blow-up formulas analogous to (4.17). The building block for the blow-up formulas is the partition function on $M=\mathbb{C}^{2}$. By the localization theorem, we obtain the result [27, 33. 43.

$$
\mathcal{Z}_{\mathbb{C}^{2}}\left(t_{1}, t_{2} ; \tau, \rho\right)=\sum_{Y} q^{|Y|} \prod_{\square \in Y}\left[\frac{\left(1-y t_{1}^{-\ell(\square)} t_{2}^{a(\square)+1}\right)\left(1-y t_{1}^{\ell(\square)+1} t_{2}^{-a(\square)}\right)}{\left(1-t_{1}^{-\ell(\square)} t_{2}^{a(\square)+1}\right)\left(1-t_{1}^{\ell(\square)+1} t_{2}^{-a(\square)}\right)}\right],
$$

where

$$
t_{1}=\mathrm{e}^{-\varepsilon_{1}} \quad \text { and } \quad t_{2}=\mathrm{e}^{-\varepsilon_{2}},
$$

while $\ell(\square)$ and $a(\square)$ are respectively the leg and arm lengths of the box $\square$ of the Young diagram $Y$. For $y=1(\rho=0)$, this agrees with the expected result (4.18). In the general case, we can sum the series (4.19) explicitly by using standard calculations on symmetric product orbifolds.

Complex plane. The Hilbert-Chow morphism $\pi: M^{[n]} \rightarrow \mathfrak{S}^{n} M$ realises the Hilbert scheme $M^{[n]}$ of $M=\mathbb{C}^{2}$ as a semi-small smooth resolution of the $n$-th symmetric product $\mathfrak{S}^{n} M$; the torus $T$ acts diagonally on $\mathfrak{S}^{n} M$. This identifies the equivariant $\chi_{y^{-}}$genera 43 ]

$$
\chi_{y}\left(\left(\mathbb{C}^{2}\right)^{[n]}\right)\left(t_{1}, t_{2}\right)=\chi_{y}\left(\mathfrak{S}^{n} \mathbb{C}^{2}\right)\left(t_{1}, t_{2}\right)
$$

where the $\chi_{y}$-genus on the right-hand side is the equivariant orbifold $\chi_{y}$-genus defined in 43 , Sect. 3]. The localization formula applied to (2.10) with $X=\mathbb{C}^{2}$ yields

$$
\chi_{y}\left(\mathbb{C}^{2}\right)\left(t_{1}, t_{2}\right)=\frac{\left(1-y t_{1}\right)\left(1-y t_{2}\right)}{\left(1-t_{1}\right)\left(1-t_{2}\right)}
$$


and therefore [43, Sect. 5.2]

$$
\begin{aligned}
\mathcal{Z}_{\mathbb{C}^{2}}\left(t_{1}, t_{2} ; \tau, \rho\right) & =\exp \left(\sum_{n=1}^{\infty} \frac{q^{n}}{n} \frac{\chi_{y^{n}}\left(\mathbb{C}^{2}\right)\left(t_{1}^{n}, t_{2}^{n}\right)}{1-y^{n} q^{n}}\right) \\
& =\prod_{n=1}^{\infty} \prod_{m_{1}, m_{2}=0}^{\infty}\left[\frac{\left(1-y^{n} q^{n} t_{1}^{m_{1}+1} t_{2}^{m_{2}}\right)\left(1-y^{n} q^{n} t_{1}^{m_{1}} t_{2}^{m_{2}+1}\right)}{\left(1-y^{n-1} q^{n} t_{1}^{m_{1}} t_{2}^{m_{2}}\right)\left(1-y^{n+1} q^{n} t_{1}^{m_{1}+1} t_{2}^{m_{2}+1}\right)}\right] \\
& =\prod_{m_{1}, m_{2}=0}^{\infty}\left[\frac{\mathcal{R}\left(\widehat{s}=\left(2 \pi \mathrm{i}(\tau+\rho)+\xi_{1,0}^{m_{1}, m_{2}}\right)(1-\mathrm{i} \varrho(\tau))-2 \pi \mathrm{i} \rho\right)}{\mathcal{R}\left(\widehat{s}=\left(2 \pi \mathrm{i} \tau+\xi_{0,0}^{m_{1}, m_{2}}\right)(1-\mathrm{i} \varrho(\tau))-2 \pi \mathrm{i} \rho\right)}\right] \\
& \times\left[\frac{\mathcal{R}\left(\widehat{s}=\left(2 \pi \mathrm{i}(\tau+\rho)+\xi_{0,1}^{m_{1}, m_{2}}\right)(1-\mathrm{i} \varrho(\tau))-2 \pi \mathrm{i} \rho\right)}{\mathcal{R}\left(\widehat{s}=\left(2 \pi \mathrm{i}(\tau+2 \rho)+\xi_{1,1}^{m_{1}, m_{2}}\right)(1-\mathrm{i} \varrho(\tau))-2 \pi \mathrm{i} \rho\right)}\right]
\end{aligned}
$$

where $\xi_{a, b}^{m_{1}, m_{2}}=-\left(m_{1}+a\right) \varepsilon_{1}-\left(m_{2}+b\right) \varepsilon_{2}$. Hence in the antidiagonal limit $t_{1}=t_{2}^{-1}=t=\mathrm{e}^{-\hbar}$, the blowup formula (4.17) yields

$$
\mathcal{Z}_{M}(\hbar ; \tau, z, \rho)=\Theta_{\Gamma}(\tau, z) \prod_{i=0}^{\ell} \mathcal{Z}_{\mathbb{C}^{2}}\left(t^{k l_{i}-(l+1) k_{i}}, t^{(l+1) k_{i+1}-k l_{i+1}} ; \tau, \rho\right)
$$

This matches Eq. (4.8) in the non-equivariant limit $t=1$ upon dropping the products over $m_{1}, m_{2}$ in (4.21). Note that in this case, since the geometry is toric, only the Hodge numbers $h^{j, j}(M)$ for $j=0,1,2$ are non-vanishing; in particular, all BPS D4-D2-D0 states have vanishing $\mathrm{R}$-charge $J_{0}=0$.

Line bundles on $\mathbb{P}^{1}$. Let $M$ be the total space of the holomorphic line bundle $\mathcal{O}_{\mathbb{P}^{1}}(-k) \rightarrow$ $\mathbb{P}^{1}$. In this case $l=1, \ell=1$ and $C=e_{1}=k$, so that the black hole partition function becomes

$$
\mathcal{Z}_{\mathcal{O}_{\mathbb{P}} 1(-k)}(\tau, z)=\frac{\vartheta_{3}\left(\frac{\tau}{k}, \frac{z}{k}\right)}{\hat{\eta}(\tau)^{2}}
$$

where $\vartheta_{3}(\tau, z)$ is the Jacobi elliptic function

$$
\vartheta_{3}(\tau, z)=\prod_{n=1}^{\infty}\left(1-q^{n}\right)\left(1+q^{n-1 / 2} w\right)\left(1+q^{n-1 / 2} w^{-1}\right) .
$$

The spectral function representation is given by combining (3.10) with

$$
\begin{aligned}
\vartheta_{3}(\tau, z) & =\mathcal{R}(\widehat{s}=2 \pi \mathrm{i} \tau(1-\mathrm{i} \varrho(\tau))) \mathcal{R}(\widehat{s}=\pi \mathrm{i}(2 z-\tau)(1-\mathrm{i} \varrho(\tau))-\mathrm{i} \widehat{\varrho}(\tau)) \\
& \times \mathcal{R}(\widehat{s}=-\pi \mathrm{i}(2 z+\tau)(1-\mathrm{i} \varrho(\tau))-\mathrm{i} \varrho(\tau)),
\end{aligned}
$$

where $\widehat{\varrho}(\tau)=\pi(\varrho(\tau)+\mathrm{i})$. The tangent weights are given by

$$
\varepsilon_{1}^{(0)}=k \varepsilon_{1}, \varepsilon_{2}^{(0)}=\varepsilon_{2}-\varepsilon_{1} \quad \text { and } \quad \varepsilon_{1}^{(1)}=\varepsilon_{1}-\varepsilon_{2}, \varepsilon_{2}^{(1)}=k \varepsilon_{2},
$$


and the refined black hole partition function is

$$
\mathcal{Z}_{\mathcal{O}_{\mathbb{P}^{1}}(-k)}(\hbar ; \tau, z, \rho)=\vartheta_{3}\left(\frac{\tau}{k}, \frac{z}{k}\right) \mathcal{Z}_{\mathbb{C}^{2}}\left(t^{k}, t^{-2} ; \tau, \rho\right) \mathcal{Z}_{\mathbb{C}^{2}}\left(\bar{t}^{k}, \bar{t}^{-2} ; \tau, \rho\right)
$$

where $\bar{t}=t^{-1}$; its spectral function representation follows from (4.21) and (4.25).

ALE spaces. The minimal toric Calabi-Yau resolution of $\mathbb{C}^{2} / \mathbb{Z}_{k}$ is the ALE space of type $A_{k-1}$. In this case $l=k-1, \ell=k$ and $e_{i}=2$ for all $i=1, \ldots, k$, so that the intersection matrix coincides with minus the Cartan matrix of the $A_{k-1}$ Dynkin diagram. The black hole partition function $\mathcal{Z}_{A_{k-1}}(\tau, z)$ is then the character of the two-dimensional conformal field theory based on the affine Lie algebra $\widehat{\mathfrak{h}} \oplus \widehat{\mathfrak{s l}}(k)_{1}\left[52\right.$; the level one Kac-Moody algebra $\widehat{\mathfrak{s l}}(k)_{1}$ is derived from the Heisenberg algebra $\widehat{\mathfrak{h}}(\Gamma)$ via the Frenkel-Kac construction. The fact that the generalised chiral algebra is a Kac-Moody algebra in this case is a consequence of the McKay correspondence. The tangent weights are given by

$$
\varepsilon_{1}^{(i)}=(k-i) \varepsilon_{1}-i \varepsilon_{2} \quad \text { and } \quad \varepsilon_{2}^{(i)}=(-k+i+1) \varepsilon_{1}+(i+1) \varepsilon_{2}
$$

for $i=0,1, \ldots, k-1$, and the refined black hole partition function is

$$
\begin{aligned}
\mathcal{Z}_{A_{k-1}}(\hbar ; \tau, z, \rho) & =\Theta_{\Gamma}(\tau, z) \prod_{n, m=0}^{\infty}\left[\frac{\left(1-q^{n+1} y^{n+1} t^{k m}\right)\left(1-q^{n+1} y^{n+1} t^{k(m+2)}\right)}{\left(1-q^{n+1} y^{n} t^{k(m+1)}\right)\left(1-q^{n+1} y^{n+2} t^{k(m+1)}\right)}\right]^{k(m+1)} \\
& =\Theta_{\Gamma}(\tau, z) \prod_{m=0}^{\infty}\left[\frac{\mathcal{R}\left(\widehat{s}=\left(2 \pi \mathrm{i}(\tau+\rho)+\xi_{0}^{m}\right)(1-\mathrm{i} \varrho(\tau))-2 \pi \mathrm{i} \rho\right)}{\mathcal{R}\left(\widehat{s}=\left(2 \pi \mathrm{i} \tau+\xi_{1}^{m}\right)(1-\mathrm{i} \varrho(\tau))-2 \pi \mathrm{i} \rho\right)}\right. \\
& \left.\times \frac{\mathcal{R}\left(\widehat{s}=\left(2 \pi \mathrm{i}(\tau+\rho)+\xi_{2}^{m}\right)(1-\mathrm{i} \varrho(\tau))-2 \pi \mathrm{i} \rho\right)}{\mathcal{R}\left(\widehat{s}=\left(2 \pi \mathrm{i}(\tau+2 \rho)+\xi_{1}^{m}\right)(1-\mathrm{i} \varrho(\tau))-2 \pi \mathrm{i} \rho\right)}\right]^{k(m+1)}
\end{aligned}
$$

where $\xi_{a}^{m}=-k(m+a) \hbar$. This is the spin character of the two-dimensional conformal field theory based on the affine Lie algebra $\widehat{\mathfrak{h}} \oplus \widehat{\mathfrak{s l}}(k)_{1}$; for $k=2$ it coincides with the partition function (4.26).

\section{$4.6(2,2)$ field theory elliptic genus}

We now consider microstates of quantum black holes that are enumerated by elliptic genera of two-dimensional superconformal field theories with $(2,2)$ worldsheet supersymmetry. Underlying all of our black hole partition functions is the elliptic genus of the D-brane moduli space $\mathcal{M}=\mathcal{M}_{n, u}(M)$ which counts supersymmetric bound states of D4-D2-D0 systems on a generic Calabi-Yau threefold. It is given by the partition function in the Ramond sector of a two-dimensional $\mathcal{N}=2$ superconformal sigma-model on the elliptic curve $\mathcal{E}$ with target manifold $\mathcal{M}$. The equivariant elliptic genus is then defined as a trace over the Hilbert space $\mathcal{H}_{n, u}(M)$ in the Ramond sector as

$$
\chi\left(\mathcal{M}_{n, u}(M) ; y, p\right)\left(t_{1}, t_{2}\right)=\operatorname{Tr}_{\mathcal{H}_{n, u}(M)}(-1)^{F_{L}} y^{J_{0}} p^{L_{0}} t_{1}^{K_{1}} t_{2}^{K_{2}},
$$


where $\left(K_{1}, K_{2}\right)$ are the generators of the abelian subgroup $T=U(1) \times U(1) \subset S U(2)_{L} \times$ $S U(2)_{R}$ of the Lorentz group of $\mathbb{R}^{4}$; the extra fugacities $t_{1}, t_{2}$ are inserted to further resolve degeneracies of states. The corresponding partition function is then given by the generating function for elliptic genera

$$
\mathcal{Z}_{M}\left(t_{1}, t_{2} ; \tau, z, \rho, \sigma\right)=\sum_{n=0}^{\infty} \sum_{u \in \Gamma} \chi\left(\mathcal{M}_{n, u}(M) ; y, p\right)\left(t_{1}, t_{2}\right) q^{n} \prod_{a=1}^{b_{2}^{+}(M)} w_{a}^{u^{a}}
$$

where $p=: \mathrm{e}^{2 \pi \mathrm{i} \sigma}$. In the non-equivariant case $t_{1}=t_{2}=1$, the elliptic genus is the supersymmetric index given in terms of multiplicative characteristic classes associated to formal power series via Eq. (2.7) with $X=\mathcal{M}_{n, u}(M), d=2\left(n-n_{u}\right)$ and $q=p$; once again we can explicitly perform the sum over instanton charges in (4.29) in this instance using results from Sect. 2.3 and Eq. (2.19) to get

$$
\mathcal{Z}_{M}(\tau, z, \rho, \sigma)=\Theta_{\Gamma}(\tau, z) \prod_{m \geq 0, l} \prod_{n=1}^{\infty}\left(1-y^{l} p^{m} q^{n}\right)^{-\kappa(m n, l)}
$$

where $\chi(M ; p, y)=\sum_{m \geq 0, l} \kappa(m, l) p^{m} y^{l}$ is the elliptic genus of $M$. The equivariant version of (4.30) follows by applying the localization theorem to the integration in (2.7). For $p=0$ it reduces to the Hirzebruch $\chi_{y}$-genus via (2.8).

To apply the blow-up formulas in this case, we first compute the partition function (4.29) on $M=\mathbb{C}^{2}$ using the localization formula to get [27, 33, 43]

$$
\begin{aligned}
\mathcal{Z}_{\mathbb{C}^{2}}\left(t_{1}, t_{2} ; \tau, \rho, \sigma\right) & =\sum_{Y}\left(y^{-1} q\right)^{|Y|} \\
& \times \prod_{\square \in Y} \prod_{m=1}^{\infty}\left[\frac{\left(1-y p^{m-1} t_{1}^{-\ell(\square)} t_{2}^{a(\square)+1}\right)\left(1-y^{-1} p^{m} t_{1}^{\ell(\square)} t_{2}^{-a(\square)-1}\right)}{\left(1-p^{m-1} t_{1}^{-\ell(\square)} t_{2}^{a(\square)+1}\right)\left(1-p^{m} t_{1}^{\ell(\square)} t_{2}^{-a(\square)-1}\right)}\right. \\
& \left.\times \frac{\left(1-y p^{m-1} t_{1}^{\ell(\square)+1} t_{2}^{-a(\square)}\right)\left(1-y^{-1} p^{m} t_{1}^{-\ell(\square)-1} t_{2}^{a(\square)}\right)}{\left(1-p^{m-1} t_{1}^{\ell(\square)+1} t_{2}^{-a(\square)}\right)\left(1-p^{m} t_{1}^{-\ell(\square)-1} t_{2}^{a(\square)}\right)}\right] .
\end{aligned}
$$

Equivalently, by applying the localization formula to the spectral function representation (3.11) we have

$$
\begin{aligned}
\mathcal{Z}_{\mathbb{C}^{2}}\left(t_{1}, t_{2} ; \tau, \rho, \sigma\right) & =\sum_{Y}\left(y^{-1} q\right)^{|Y|} \prod_{\square \in Y}\left[\frac{\mathcal{R}\left(\widehat{s}=\left(2 \pi \mathrm{i} \rho+\xi_{-\ell(\square), a(\square)+1}^{0,0}\right)(1-\mathrm{i} \varrho(\sigma))\right)}{\mathcal{R}\left(\widehat{s}=\xi_{-\ell(\square), a(\square)+1}^{0,0}(1-\mathrm{i} \varrho(\sigma))\right)}\right] \\
& \times\left[\frac{\mathcal{R}\left(\widehat{s}=\left(2 \pi \mathrm{i}(\sigma-\rho)+\xi_{\ell(\square),-a(\square)-1}^{0,0}\right)(1-\mathrm{i} \varrho(\sigma))\right)}{\mathcal{R}\left(\widehat{s}=\left(2 \pi \mathrm{i} \sigma+\xi_{\ell(\square),-a(\square)-1}^{0,0}\right)(1-\mathrm{i} \varrho(\sigma))\right)}\right] \\
& \times\left[\frac{\mathcal{R}\left(\widehat{s}=\left(2 \pi \mathrm{i} \rho+\xi_{\ell(\square)+1,-a(\square)}^{0,0}\right)(1-\mathrm{i} \varrho(\sigma))\right)}{\mathcal{R}\left(\widehat{s}=\xi_{\ell(\square)+1,-a(\square)}^{0,0}(1-\mathrm{i} \varrho(\tau))\right)}\right] \\
& \times\left[\frac{\mathcal{R}\left(\widehat{s}=\left(2 \pi \mathrm{i}(\sigma-\rho)+\xi_{-\ell(\square)-1, a(\square)}^{0,0}\right)(1-\mathrm{i} \varrho(\sigma))\right)}{\mathcal{R}\left(\widehat{s}=\left(2 \pi \mathrm{i} \sigma+\xi_{-\ell(\square)-1, a(\square)}^{0,0}\right)(1-\mathrm{i} \varrho(\sigma))\right)}\right] .
\end{aligned}
$$


Following [19, 13, 43], we can sum this series explicitly by identifying this partition function with the generating function for orbifold equivariant elliptic genera of symmetric products given by

$$
\mathcal{Z}_{\mathbb{C}^{2}}\left(t_{1}, t_{2} ; \tau, \rho, \sigma\right)=\sum_{n=0}^{\infty} \chi\left(\mathfrak{S}^{n} \mathbb{C}^{2} ; y, p\right)\left(t_{1}, t_{2}\right) q^{n}
$$

The localization formula applied to (2.7) with $X=\mathbb{C}^{2}$ and $q=p$ yields

$$
\begin{aligned}
\chi\left(\mathbb{C}^{2} ; y, p\right)\left(t_{1}, t_{2}\right) & =y^{-1} \frac{\left(1-y t_{1}\right)\left(1-y t_{2}\right)}{\left(1-t_{1}\right)\left(1-t_{2}\right)} \\
& \times \prod_{m=1}^{\infty}\left[\frac{\left(1-y p^{m} t_{1}\right)\left(1-y^{-1} p^{m} t_{1}^{-1}\right)\left(1-y p^{m} t_{2}\right)\left(1-y^{-1} p^{m} t_{2}^{-1}\right)}{\left(1-p^{m} t_{1}\right)\left(1-p^{m} t_{1}^{-1}\right)\left(1-p^{m} t_{2}\right)\left(1-p^{m} t_{2}^{-1}\right)}\right] \\
& =y^{-1} \frac{\left(1-y t_{1}\right)\left(1-y t_{2}\right)}{\left(1-t_{1}\right)\left(1-t_{2}\right)}\left[\frac{\mathcal{R}\left(\widehat{s}=\left(2 \pi \mathrm{i}(\sigma+\rho)-\varepsilon_{1}\right)(1-\mathrm{i} \varrho(\sigma))\right)}{\mathcal{R}\left(\widehat{s}=\left(2 \pi \mathrm{i} \sigma-\varepsilon_{1}\right)(1-\mathrm{i} \varrho(\sigma))\right)}\right] \\
& \times\left[\frac{\mathcal{R}\left(\widehat{s}=\left(2 \pi \mathrm{i}(\sigma-\rho)+\varepsilon_{1}\right)(1-\mathrm{i} \varrho(\sigma))\right)}{\mathcal{R}\left(\widehat{s}=\left(2 \pi \mathrm{i} \sigma+\varepsilon_{1}\right)(1-\mathrm{i} \varrho(\sigma))\right)}\right] \\
& \times\left[\frac{\mathcal{R}\left(\widehat{s}=\left(2 \pi \mathrm{i}(\sigma+\rho)-\varepsilon_{2}\right)(1-\mathrm{i} \varrho(\sigma))\right)}{\mathcal{R}\left(\widehat{s}=\left(2 \pi \mathrm{i} \sigma-\varepsilon_{2}\right)(1-\mathrm{i} \varrho(\sigma))\right)}\right] \\
& \times\left[\frac{\mathcal{R}\left(\widehat{s}=\left(2 \pi \mathrm{i}(\sigma-\rho)+\varepsilon_{2}\right)(1-\mathrm{i} \varrho(\sigma))\right)}{\mathcal{R}\left(\widehat{s}=\left(2 \pi \mathrm{i} \sigma+\varepsilon_{2}\right)(1-\mathrm{i} \varrho(\sigma))\right)}\right] .
\end{aligned}
$$

By [43, Thm. 3.1] one then has

$$
\mathcal{Z}_{\mathbb{C}^{2}}\left(t_{1}, t_{2} ; \tau, \rho, \sigma\right)=\exp \left(\sum_{n, n^{\prime}=1}^{\infty} \frac{q^{n n^{\prime}}}{n^{\prime}} \frac{1}{n} \sum_{j=0}^{n-1} \chi\left(\mathbb{C}^{2} ; y^{n^{\prime}}, \mathrm{e}^{2 \pi \mathrm{i} j n^{\prime} / n} p^{n^{\prime} / n}\right)\left(t_{1}^{n^{\prime}}, t_{2}^{n^{\prime}}\right)\right)
$$

Let us work out the corresponding free energy. For this, we define integers $\kappa\left(l, m, k_{1}, k_{2}\right) \in \mathbb{Z}$ by the expansion

$$
\begin{aligned}
& \prod_{m=1}^{\infty}\left[\frac{\left(1-y p^{m} t_{1}\right)\left(1-y^{-1} p^{m} t_{1}^{-1}\right)\left(1-y p^{m} t_{2}\right)\left(1-y^{-1} p^{m} t_{2}^{-1}\right)}{\left(1-p^{m} t_{1}\right)\left(1-p^{m} t_{1}^{-1}\right)\left(1-p^{m} t_{2}\right)\left(1-p^{m} t_{2}^{-1}\right)}\right] \\
= & \sum_{l=0}^{\infty} \sum_{m, k_{1}, k_{2} \in \mathbb{Z}} \kappa\left(l, m, k_{1}, k_{2}\right) p^{l} y^{m} t_{1}^{k_{1}} t_{2}^{k_{2}} .
\end{aligned}
$$

Since the left-hand side of (4.35) is invariant under the changes of variables $t_{1} \leftrightarrow t_{2}$ and $y \rightarrow y^{-1}, t_{1} \rightarrow t_{1}^{-1}, t_{2} \rightarrow t_{2}^{-1}$, these integers have the symmetry properties

$$
\kappa\left(l, m, k_{1}, k_{2}\right)=\kappa\left(l, m, k_{2}, k_{1}\right)=\kappa\left(l,-m,-k_{1},-k_{2}\right) .
$$


We thus have

$$
\begin{aligned}
\log \mathcal{Z}_{\mathbb{C}^{2}}\left(t_{1}, t_{2} ; \tau, \rho, \sigma\right) & =\sum_{n, n^{\prime}=1}^{\infty} \frac{\left(y^{-1} q^{n}\right)^{n^{\prime}}}{n^{\prime}} \frac{\left(1-y^{n^{\prime}} t_{1}^{n^{\prime}}\right)\left(1-y^{n^{\prime}} t_{2}^{n^{\prime}}\right)}{\left(1-t_{1}^{n^{\prime}}\right)\left(1-t_{2}^{n^{\prime}}\right)} \\
& \times \frac{1}{n} \sum_{j=0}^{n-1} \sum_{l=0}^{\infty} \sum_{m, k_{1}, k_{2} \in \mathbb{Z}} \kappa\left(l, m, k_{1}, k_{2}\right) \mathrm{e}^{2 \pi \mathrm{i} l j / n} p^{l n^{\prime} / n} y^{m n^{\prime}} t_{1}^{k_{1} n^{\prime}} t_{2}^{k_{2} n^{\prime}} \\
& =\sum_{n, n^{\prime}=1}^{\infty} \frac{\left(y^{-1} q^{n}\right)^{n^{\prime}}}{n^{\prime}}\left(1-y^{n^{\prime}} t_{1}^{n^{\prime}}-y^{n^{\prime}} t_{2}^{n^{\prime}}+y^{2 n^{\prime}} t_{1}^{n^{\prime}} t_{2}^{n^{\prime}}\right) \sum_{r_{1}, r_{2}=0}^{\infty} t_{1}^{n^{\prime} r_{1}} t_{2}^{n^{\prime} r_{2}} \\
& \times \sum_{l=0}^{\infty} \sum_{m, k_{1}, k_{2} \in \mathbb{Z}} \kappa\left(n l, m, k_{1}, k_{2}\right) p^{l n^{\prime}} y^{m n^{\prime}} t_{1}^{k_{1} n^{\prime}} t_{2}^{k_{2} n^{\prime}} \\
& =-\sum_{l, r_{1}, r_{2}=0}^{\infty} \sum_{m, k_{1}, k_{2} \in \mathbb{Z}} \sum_{n=1}^{\infty} \kappa\left(n l, m, k_{1}, k_{2}\right) \\
& \times \log \left[\left(\frac{1-q^{n} y^{m-1} p^{l} t_{1}^{k_{1}+r_{1}} t_{2}^{k_{2}+r_{2}}}{1-q^{n} y^{m} p^{l} t_{1}^{k_{1}+r_{1}+1} t_{2}^{k_{2}+r_{2}}}\right)\right. \\
& \left.\times\left(\frac{1-q^{n} y^{m+1} p^{l} t_{1}^{k_{1}+r_{1}+1} t_{2}^{k_{2}+r_{2}+1}}{1-q^{n} y^{m} p^{l} t_{1}^{k_{1}+r_{1}} t_{2}^{k_{2}+r_{2}+1}}\right)\right]
\end{aligned}
$$

and we finally get

$$
\begin{aligned}
\mathcal{Z}_{\mathbb{C}^{2}}\left(t_{1}, t_{2} ; \tau, \rho, \sigma\right) & =\prod_{\substack{l, r_{1}, r_{2} \geq 0, n>0 \\
m, k_{1}, k_{2} \in \mathbb{Z}}}\left[\left(\frac{1-y^{m} p^{l} q^{n} t_{1}^{k_{1}+r_{1}+1} t_{2}^{k_{2}+r_{2}}}{1-y^{m-1} p^{l} q^{n} t_{1}^{k_{1}+r_{1}} t_{2}^{k_{2}+r_{2}}}\right)\right. \\
& \left.\times\left(\frac{1-y^{m} p^{l} q^{n} t_{1}^{k_{1}+r_{1}} t_{2}^{k_{2}+r_{2}+1}}{1-y^{m+1} p^{l} q^{n} t_{1}^{k_{1}+r_{1}+1} t_{2}^{k_{2}+r_{2}+1}}\right)\right]^{\kappa\left(n l, m, k_{1}, k_{2}\right)} .
\end{aligned}
$$

The blow-up formula (4.17) in the antidiagonal limit yields

$$
\mathcal{Z}_{M}(\hbar ; \tau, z, \rho, \sigma)=\Theta_{\Gamma}(\tau, z) \prod_{i=0}^{\ell} \mathcal{Z}_{\mathbb{C}^{2}}\left(t^{k l_{i}-(l+1) k_{i}}, t^{(l+1) k_{i+1}-k l_{i+1}} ; \tau, \rho, \sigma\right) .
$$

For example, when $M$ is the total space of the holomorphic line bundle $\mathcal{O}_{\mathbb{P}^{1}}(-k) \rightarrow \mathbb{P}^{1}$, we get

$$
\mathcal{Z}_{\mathcal{O}_{\mathbb{P} 1}(-k)}(\hbar ; \tau, z, \rho, \sigma)=\vartheta_{3}\left(\frac{\tau}{k}, \frac{z}{k}\right) \mathcal{Z}_{\mathbb{C}^{2}}\left(t^{k}, t^{-2} ; \tau, \rho, \sigma\right) \mathcal{Z}_{\mathbb{C}^{2}}\left(\bar{t}^{k}, \bar{t}^{-2} ; \tau, \rho, \sigma\right) .
$$

On the other hand, when $M$ is the ALE space of type $A_{k-1}$ we find

$$
\begin{aligned}
\mathcal{Z}_{A_{k-1}}(\hbar ; \tau, z, \rho, \sigma) & =\Theta_{\Gamma}(\tau, z) \prod_{\substack{l, r \geq 0, n>0 \\
m, j \in \mathbb{Z}}}\left[\left(\frac{1-y^{m} p^{l} q^{n} t^{k(j+1)}}{1-y^{m-1} p^{l} q^{n} t^{k j}}\right)\right. \\
& \left.\times\left(\frac{1-y^{m} p^{l} q^{n} t^{k(j-1)}}{1-y^{m+1} p^{l} q^{n} t^{k j}}\right)\right]^{k(r+1) \kappa(n l, m, j-r)}
\end{aligned}
$$


where $\kappa(n, m, j):=\kappa\left(n, m, k_{1}-k_{2}, 0\right)$ in the expansion (4.35) with $t_{1}=t_{2}^{-1}=t$.

The elliptic genera (4.37) and (4.40) can be described in terms of a set of Kerov's symmetric functions [37]. Using the Cauchy identity for Kerov's functions in the Hall-Littlewood case and Ruelle-type spectral functions, one can obtain the corresponding spectral partition functions in their final form analogously to our M-theory partition functions. Similar calculations exist in the literature for the matrix elements of the currents of the quantum affine algebra $U_{q}(\widehat{\mathfrak{s l}}(2))$ (see e.g. [38]). The derivations and resulting formulas are somewhat cumbersome to describe and lie outside the scope of the present paper; we plan to address this problem elsewhere.

As we show in Appendix B, the elliptic genus can be continued to an entire function without essential singularities. It would be interesting to understand its relation to the elliptic genus of the $(4,0)$ superconformal field theory in the M-theory frame. This presumably entails constructing partition functions which depend on moduli which measure fluctuations of the D4-brane around the divisor class of its worldvolume $M$ in $X$, and similarly for the D2-branes; in this region of the moduli space one must study the full nonlinear Dirac-Born-Infeld theory as the worldvolume effective theory, and the connected components of the D-brane moduli space are expected to be singular fibrations over the Hilbert schemes $M^{[n]}$. Then one should find a sequence of duality transformations to a particular chamber of the moduli space where the D4-brane decays into a D6 brane-antibrane pair, with the D6-brane worldvolume theory governed by a suitable refinement or categorification of Donaldson-Thomas theory (or equivalently topological string theory) as in [1] ; via this chain of dualities the $A d S_{2} \times S^{2} \times X$ near horizon geometry is related to Type IIA string theory on $X$ with a D6 brane-antibrane pair, where the M-theory lift of the D6-brane is a Taub-NUT geometry.

Note that such walls of marginal stability can in principal arise by computing degeneracies from Fourier coefficients of the elliptic genus as in Eq. (3.7); the moduli dependence is due to the moduli dependence of the Fourier integration contours and the pole structure of the partition functions described in Appendix B. Moving around the moduli space corresponds to deforming the contour and crossing a wall in the moduli space corresponds to crossing a pole of the partition function. The jump in the degeneracy upon crossing a wall from one domain to another is given by the residue at the pole which is crossed, as computed in Appendix B, It would be interesting to see if the equivariant deformation of the elliptic genera computed here could be used in this way to reproduce our M-theory partition functions by analysing the pole structures in the deformation parameters $\left(t_{1}, t_{2}\right)$.

\section{Conclusions}

In this paper we discussed various applications of affine Lie algebra representations, Hilbert schemes, elliptic genera and their generalizations to the computation of M-theory and Dbrane quantum partition functions for microscopic black hole ensembles. A central concept in all examples considered in this paper is that of elliptic genera. The notion of elliptic genus was introduced in [48] with applications to quantum field theory in [55]. It has been argued that it is possible to use elliptic modular forms to write generating functions of 
quantum field theory as infinite series of operators associated with homologies of finitedimensional Lie algebras. The elliptic genus can be interpreted as a natural invariant in a generalized cohomology theory, called elliptic cohomology [40, 41], which can be regarded as a extension of K-theory. From this point of view these partition functions might be related to elliptic cohomology and K-theory. All examples considered in this paper point towards this conjectural link. We have shown that elliptic genera, in the context of black hole partition functions, can be converted into products of spectral functions associated with $q$-series which inherit the (co)homology properties of appropriate (poly)graded Lie algebras. This common feature, that quantum generating functions can be reproduced in terms of spectral functions, encodes the connection with infinite-dimensional Lie algebras and their homologies, together with the remarkable link to hyperbolic geometry.

\section{Acknowledgments}

AAB would like to acknowledge the Conselho Nacional de Desenvolvimento Científico e Tecnológico (CNPq, Brazil) and Fundaçao Araucaria (Parana, Brazil) for financial support. The support of the Academy of Finland under the Projects No. 136539 and 140886 is gratefully acknowledged. The work of RJS was partially supported by the Consolidated Grant ST/J000310/1 from the UK Science and Technology Facilities Council, and by Grant RPG-404 from the Leverhulme Trust.

\section{A Spectral functions of hyperbolic three-manifolds}

The Euclidean sector of $A d S_{3}$ has a quotient description as a closed oriented hyperbolic threemanifold $\mathbb{H}^{3} / \mathfrak{G}^{\gamma}$. The complex unimodular group $G=S L(2, \mathbb{C})$ acts on the real hyperbolic three-space $\mathbb{H}^{3}=\left\{(x, y, z) \in \mathbb{R}^{3} \mid z>0\right\}$ in the standard way: for $(x, y, z) \in \mathbb{H}^{3}$ and

$$
g=\left(\begin{array}{ll}
a & b \\
c & d
\end{array}\right) \in G
$$

one has $g \triangleright(x, y, z)=(u, v, w) \in \mathbb{H}^{3}$, where

$$
u+\mathrm{i} v=\frac{(a r+b) \overline{(c r+d)}+a \bar{c} z^{2}}{|c r+d|^{2}+|c|^{2} z^{2}} \quad \text { and } \quad w=\frac{z}{|c r+d|^{2}+|c|^{2} z^{2}},
$$

with $r=x+$ i $y$. Let $\mathfrak{G}^{\gamma} \subset G$ be the infinite discrete Schottky subgroup of $G$ defined as

$$
\mathfrak{G}^{\gamma}=\left\{\gamma^{n} \mid n \in \mathbb{Z}\right\}, \quad \text { with } \quad \gamma=\operatorname{diag}\left(\mathrm{e}^{2 \pi \mathrm{i} \bar{\tau}}, \mathrm{e}^{-2 \pi \mathrm{i} \bar{\tau}}\right)
$$

for $\tau \in \mathbb{C}^{+}$; this subgroup acts on $\mathbb{H}^{3}$ as a rotation through angle $2 \pi \operatorname{Re} \tau$ in the $(x, y)$-plane and a dilatation by $\mathrm{e}^{2 \pi \operatorname{Im} \tau}$.

One can construct a Selberg-type zeta-function for any subgroup $\mathfrak{G}^{\gamma}:=\mathfrak{G}_{(a, b)}^{\gamma}$ generated by a single hyperbolic element of the form $\gamma:=\gamma_{(a, b)}=\operatorname{diag}\left(\mathrm{e}^{z}, \mathrm{e}^{-z}\right)$, where $z=a+\mathrm{i} b$ with $a, b>0$; for the case of $A d S_{3}$ we specialise to $a=2 \pi \operatorname{Im} \tau$ and $b=2 \pi \operatorname{Re} \tau$. Then 
the Patterson-Selberg spectral function $Z_{\mathfrak{G} \gamma}(s)$ which can be attached to the hyperbolic three-manifold $\mathbb{H}^{3} / \mathfrak{G}^{\gamma}$ (with acyclic orthogonal representation of $\pi_{1}\left(\mathbb{H}^{3} / \mathfrak{G}^{\gamma}\right)$ ) has the form

$$
Z_{\mathfrak{G} \gamma}(s):=\prod_{k_{1}, k_{2}=0}^{\infty}\left(1-\mathrm{e}^{\mathrm{i} b\left(k_{1}-k_{2}\right)} \mathrm{e}^{-a\left(k_{1}+k_{2}+s\right)}\right) .
$$

The zeroes of $Z_{\mathfrak{G} \gamma}(s)$ are precisely the set of complex numbers

$$
\zeta_{n, k_{1}, k_{2}}=-\left(k_{1}+k_{2}\right)+\mathrm{i}\left(k_{1}-k_{2}\right) \frac{b}{a}+\frac{2 \pi \mathrm{i} n}{a},
$$

with $n \in \mathbb{Z}$. The magnitude of the zeta-function is bounded for both $\operatorname{Re} s \geq 0$ and $\operatorname{Re} s \leq 0$, and its growth can be estimated as

$$
\left|Z_{\mathfrak{G} \gamma}(s)\right| \leq\left(\prod_{k_{1}+k_{2} \leq|s|} \mathrm{e}^{|s| \ell}\right)\left(\prod_{k_{1}+k_{2} \geq|s|}\left(1-\mathrm{e}^{\left(|s|-k_{1}-k_{2}\right) \ell}\right)\right) \leq C_{1} \mathrm{e}^{C_{2}|s|^{3}}
$$

for suitable constants $\ell, C_{1}, C_{2}$. The first product on the right-hand side of (A.3) gives the exponential growth, while the second product is bounded. The spectral function $Z_{\mathfrak{G} \gamma}(s)$ is an entire function of order three and of finite type which can be written as a Hadamard product [50]

$$
Z_{\mathfrak{G} \gamma}(s)=\mathrm{e}^{Q(s)} \prod_{\zeta \in \Sigma}\left(1-\frac{s}{\zeta}\right) \exp \left(\frac{s}{\zeta}+\frac{s^{2}}{2 \zeta^{2}}+\frac{s^{3}}{3 \zeta^{3}}\right),
$$

where $\Sigma$ is the set of zeroes $\zeta:=\zeta_{n, k_{1}, k_{2}}$ and $Q(s)$ is a polynomial of degree at most three. From the Hadamard product representation (A.4) it follows that

$$
\frac{\mathrm{d}}{\mathrm{d} s} \log Z_{\mathfrak{G} \gamma}(s)=\frac{\mathrm{d}}{\mathrm{d} s} Q(s)+\sum_{\zeta \in \Sigma} \frac{(s / \zeta)^{3}}{s-\zeta} .
$$

Let us define $\Xi(y \pm \mathrm{i} \xi):=\frac{\mathrm{d}}{\mathrm{d} s} \log Z_{\mathfrak{G} \gamma}(s)$ for $s=y \pm \mathrm{i} \xi$. Then

$$
\Xi(y \pm \mathrm{i} \xi)=\frac{\mathrm{d}}{\mathrm{d} s} Q(s=y \pm \mathrm{i} \xi)-\mathrm{i} \sum_{y \pm \mathrm{i} \varepsilon \in \Sigma} \frac{(y \pm \mathrm{i} \xi)^{3}}{(y \pm \mathrm{i} \varepsilon)^{3}( \pm \xi-\varepsilon)} .
$$

Let us introduce next the Ruelle zeta-functions $\mathcal{R}(s)$ [14, 10, 11. The function $\mathcal{R}(s)$ is a ratio of Patterson-Selberg zeta-functions given by $\mathcal{R}(s):=Z_{\mathfrak{G} \gamma}(s) Z_{\mathfrak{G} \gamma}(s+2) / Z_{\mathfrak{G} \gamma}(s+1)$; it is defined for Re $s \gg 1$ and can be continued to a meromorphic function on the entire complex plane $\mathbb{C}$. Its value $\mathcal{R}(0)$ computes the $L^{2}$-analytic torsion of the hyperbolic three-manifold $\mathbb{H}^{3} / \mathfrak{G}^{\gamma}$, and in the case of $A d S_{3}$ one has the infinite product identities:

$$
\begin{aligned}
\prod_{n=m}^{\infty}\left(1-q^{\mu n+\varepsilon}\right) & =\frac{Z_{\mathfrak{G} \gamma}(s)}{Z_{\mathfrak{G} \gamma}(s+\mu(1+\mathrm{i} \varrho(\tau)))} \\
& =\mathcal{R}(s=(\mu m+\varepsilon)(1-\mathrm{i} \varrho(\tau))+1-\mu) \\
\prod_{n=m}^{\infty}\left(1+q^{\mu n+\varepsilon}\right) & =\frac{Z_{\mathfrak{G} \gamma}(s)}{Z_{\mathfrak{G} \gamma}(s+\mu(1+\mathrm{i} \varrho(\tau)))} \\
& =\mathcal{R}(s=(\mu m+\varepsilon)(1-\mathrm{i} \varrho(\tau))+1-\mu+\mathrm{i} /(2 \operatorname{Im} \tau))
\end{aligned}
$$


where $q:=\mathrm{e}^{2 \pi \mathrm{i} \tau}, \varrho(\tau)=\operatorname{Re} \tau / \operatorname{Im} \tau, \mu \in \mathbb{R}, m \geq 1$ and $\varepsilon \in \mathbb{C}$. For $\nu \in \mathbb{C}$, we can use the Ruelle functions $\mathcal{R}(s)$ to naturally write more general infinite product identities:

$$
\begin{aligned}
\prod_{n=m}^{\infty}\left(1-q^{\mu n+\varepsilon}\right)^{\nu n} & =\mathcal{R}(s=(\mu m+\varepsilon)(1-\mathrm{i} \varrho(\tau))+1-\mu)^{\nu m} \\
& \times \prod_{n=m+1}^{\infty} \mathcal{R}(s=(\mu n+\varepsilon)(1-\mathrm{i} \varrho(\tau))+1-\mu)^{\nu}, \\
\prod_{n=m}^{\infty}\left(1+q^{\mu n+\varepsilon}\right)^{\nu n} & =\mathcal{R}(s=(\mu m+\varepsilon)(1-\mathrm{i} \varrho(\tau))+1-\mu+\mathrm{i} /(2 \operatorname{Im} \tau))^{\nu m} \\
& \times \prod_{n=m+1}^{\infty} \mathcal{R}(s=(\mu n+\varepsilon)(1-\mathrm{i} \varrho(\tau))+1-\mu+\mathrm{i} /(2 \operatorname{Im} \tau))^{\nu} .
\end{aligned}
$$

\section{B Singularities and poles of elliptic genera}

As a function of $y$, the elliptic genus $\chi(M ; q, y)$ in (2.11) has infinitely many simple poles at the values $y=q^{1-m+v}$ and $y=q^{m+v}$, where $v=c_{1}([M]) / 2 \pi \mathrm{i} \tau$ and $m$ is a positive integer. One can calculate the residues of these simple poles. For this, let us set

$$
\mathcal{P}(y):=\prod_{n=1}^{\infty} \frac{\left(1-q^{n-1-v}\right)\left(1-q^{n+v}\right)}{\left(1-y q^{n-1-v}\right)\left(1-y^{-1} q^{n+v}\right)} .
$$

Then

$$
\begin{aligned}
\operatorname{Res}\left(\mathcal{P}(y), q^{1-m+v}\right) & =\lim _{y \rightarrow q^{1-m+v}}\left(y-q^{1-m+v}\right) \mathcal{P}(y) \\
& =-q^{1-m+v} \prod_{n \neq m} \frac{1}{1-q^{n-m}} \prod_{n=1}^{\infty} \frac{\left(1-q^{n-1-v}\right)\left(1-q^{n+v}\right)}{1-q^{n+m-1}} .
\end{aligned}
$$

Note that

$$
\prod_{n \neq m} \frac{1}{1-q^{n-m}}=\prod_{n=1}^{m-1} \frac{1}{1-q^{n-m}} \prod_{n=m+1}^{\infty} \frac{1}{1-q^{n-m}}=\frac{1}{\left(q^{-m} ; q\right)_{m-1} \mathcal{R}(s=1-\mathrm{i} \varrho(\tau))}
$$

where $(a ; q)_{m}:=\prod_{j=1}^{m}\left(1-a q^{j}\right)$, and therefore

$$
\begin{aligned}
\operatorname{Res}\left(\mathcal{P}(y), q^{1-m+v}\right) & =-\frac{q^{1-m+v}}{\left(q^{-m} ; q\right)_{m-1}} \\
& \times\left[\frac{\mathcal{R}(s=-v(1-\mathrm{i} \varrho(\tau))) \mathcal{R}(s=(1+v)(1-\mathrm{i} \varrho(\tau)))}{\mathcal{R}(s=1-\mathrm{i} \varrho(\tau)) \mathcal{R}(s=m(1-\mathrm{i} \varrho(\tau)))}\right] .
\end{aligned}
$$


An easy way of calculating the residue at $y=q^{m+v}$ is to replace $v \rightarrow v-1, m \rightarrow-m$ to get

$$
\begin{aligned}
\operatorname{Res}\left(\mathcal{P}(y), q^{m+v}\right) & =-\frac{q^{m+v}}{\left(q^{m} ; q\right)_{m-1}} \\
& \times\left[\frac{\mathcal{R}(s=-v(1-\mathrm{i} \varrho(\tau))) \mathcal{R}(s=(1+v)(1-\mathrm{i} \varrho(\tau)))}{\mathcal{R}(s=(2 m+1)(1-\mathrm{i} \varrho(\tau))) \mathcal{R}(s=m(1-\mathrm{i} \varrho(\tau)))}\right] .
\end{aligned}
$$

Hence we can construct an entire function which has no essential singularities in the complex plane (except possibly at infinity). Similar pole structures are contained in the explicit formulas for all elliptic genera of Sects. 2.1, 3.4, 4.5 and 4.6. In these examples one can use a subtraction procedure to remove singularities, some elements of which have been presented here.

\section{References}

[1] M. Aganagic and K. Schaeffer, Refined black hole ensembles and topological strings, JHEP 01 (2013) 060; [arXiv:hep-th/1210.1865].

[2] A. Beauville, Variété Kähleriennes dont la premiére classe de Chern est nulle, J. Diff. Geom. 18 (1983) 755-782.

[3] K. Behrend and B. Fantechi, Symmetric obstruction theories and Hilbert schemes of points on threefolds, Algebra Number Theory 2 (2008) 313-345; arXiv:math.AG/0512556.

[4] K. Behrend, J. Bryan and B. Szendrői, Motivic degree zero Donaldson-Thomas invariants, Invent. Math. 192 (2013) 111-160; [arXiv:math.AG/0909.5088].

[5] I. Bena and P. Kraus, Three charge supertubes and black hole hair, Phys. Rev. D 70 (2004) 046003; arXiv:hep-th/0402144.

[6] I. N. Bernshtein and B. I. Rozenfel'd, Homogeneous spaces of infinite-dimensional Lie algebras and characteristic classes of foliations, Russian Math. Surveys 28 (1973) 107142.

[7] M. Bershadsky, V. Sadov and C. Vafa, D-branes and topological field theories, Nucl. Phys. B 463 (1996) 420-434; arXiv:hep-th/9511222.

[8] J. de Boer, Six-dimensional supergravity on $S^{3} \times A d S_{3}$ and $2 D$ conformal field theory, Nucl. Phys. B 548 (1999) 139-166; arXiv:hep-th/9806104.

[9] G. Bonelli, K. Maruyoshi, A. Tanzini and F. Yagi, $\mathcal{N}=2$ gauge theories on toric singularities, blow-up formulae and W-algebrae, JHEP 01 (2013) 014; [arXiv:hepth/1208.0790]. 
[10] L. Bonora and A. A. Bytsenko, Partition functions for quantum gravity, black holes, elliptic genera and Lie algebra homologies, Nucl. Phys. B 852 (2011) 508-537; [arXiv:hep-th/1105.4571].

[11] L. Bonora, A. A. Bytsenko and E. Elizalde, String partition functions, Hilbert schemes and affine Lie algebra representations on homology groups, J. Phys. A 45 (2012) 374002; [arXiv:hep-th/1206.0664].

[12] L. A. Borisov and A. Libgober, Elliptic genera of toric varieties and applications to mirror symmetry, Invent. Math. 140 (2000) 453-485; arXiv:math.AG/9904126.

[13] L. A. Borisov and A. Libgober, McKay correspondence for elliptic genera, Ann. Math. 161 (2005) 1521-1569; arXiv:math.AG/0206241.

[14] A. A. Bytsenko, M. E. X. Guimãraes and F. L. Williams, Remarks on the spectrum and truncated heat kernel of the BTZ black hole, Lett. Math. Phys. 79 (2007) 203-211; arXiv:hep-th/0609102.

[15] A. A. Bytsenko and A. Tureanu, Quantum corrections to Bekenstein-Hawking black hole entropy and gravity partition functions, Nucl. Phys. B 873 (2013) 534-549; [arXiv:hep-th/1304.7021].

[16] A. A. Bytsenko, M. Chaichian, A. Tureanu and F. L. Williams, BRST-invariant deformations of geometric structures in topological field theories, Int. J. Mod. Phys. A 28 (2013) 1350069; [arXiv:hep-th/1306.0373].

[17] M. Cirafici, A.-K. Kashani-Poor and R. J. Szabo, Crystal melting on toric surfaces, J. Geom. Phys. 61 (2011) 2199-2218; [arXiv:hep-th/0912.0737].

[18] L. S. Cirio, G. Landi and R. J. Szabo, Instantons and vortices on noncommutative toric varieties, arXiv:hep-th/1212.3469.

[19] R. Dijkgraaf, G. W. Moore, E. Verlinde and H. Verlinde, Elliptic genera of symmetric products and second quantized strings, Commun. Math. Phys. 185 (1997) 197-209; arXiv:hep-th/9608096.

[20] T. Eguchi, H. Ooguri, A. Taormina and S.-K. Yang, Superconformal algebras and string compactification on manifolds with $S U(N)$ holonomy, Nucl. Phys. B 315 (1989) $193-221$.

[21] J. Fogarty, Algebraic families on an algebraic surface, Amer. J. Math. 90 (1968) 511521.

[22] F. Fucito, J. F. Morales and R. Poghossian, Instanton on toric singularities and black hole countings, JHEP 12 (2006) 073; arXiv:hep-th/0610154.

[23] A. Fujiki, On primitively symplectic compact Kähler V-manifolds of dimension four, Progr. Math. 39 (1983) 71-250. 
[24] D. B. Fuks, Cohomology of Infinite-Dimensional Lie Algebras (Consultants Bureau, New York, 1986).

[25] D. Gaiotto, A. Strominger and X. Yin, Superconformal black hole quantum mechanics, JHEP 11 (2005) 017; arXiv:hep-th/0412322.

[26] D. Gaiotto, A. Strominger and X. Yin, From AdS $S_{3} / C F T_{2}$ to black holes/topological strings, JHEP 09 (2007) 050; hep-th/0602046.

[27] E. Gasparim and C. -C. M. Liu, The Nekrasov conjecture for toric surfaces, Commun. Math. Phys. 293 (2010) 661-700; [arXiv:math.AG/0808.0884].

[28] R. Gopakumar and C. Vafa, M-theory and topological strings. 2., arXiv:hep-th/9812127.

[29] L. Göttsche, The Betti numbers of the Hilbert scheme of points on a smooth projective surface, Math. Ann. 286 (1990) 193-207.

[30] L. Griguolo, D. Seminara, R. J. Szabo and A. Tanzini, Black holes, instanton counting on toric singularities and q-deformed two-dimensional Yang-Mills theory, Nucl. Phys. B 772 (2007) 1-24; arXiv:hep-th/0610155.

[31] P. Griffiths and J. Harris, Principles of Algebraic Geometry (John Wiley \& Sons, New York, 1978).

[32] F. Hirzebruch and T. Höfer, On the Euler number of an orbifold, Math. Ann. 286 (1990) 255-260.

[33] T. J. Hollowood, A. Iqbal and C. Vafa, Matrix models, geometric engineering and elliptic genera, JHEP 03 (2008) 069; arXiv:hep-th/0310272.

[34] S. Hosono, M.-H. Sato and A. Takahashi, Relative Lefschetz action and BPS state counting, Int. Math. Res. Notices 15 (2001) 783-816; arXiv:math.AG/0105148.

[35] S. Katz, A. Klemm and C. Vafa, M-theory, topological strings and spinning black holes, Adv. Theor. Math. Phys. 3 (1999) 1445-1537; arXiv:hep-th/9910181].

[36] T. Kawai, Y. Yamada and S.-K. Yang, Elliptic genera and $\mathcal{N}=2$ superconformal field theory, Nucl. Phys. B 414 (1994) 191-212; arXiv:hep-th/9306096].

[37] S. V. Kerov, Hall-Littlewood functions and orthogonal polynomials, Funct. Anal. Appl. 25 (1991) 65-66.

[38] R. C. King, Symmetric functions and characters of Lie algebras and superalgebras, in: Invariant Theory and Tableaux, D. Stanton (Ed.) (Springer, New York, 1990) 226-261.

[39] P. Kraus and F. Larsen, Partition functions and elliptic genera from supergravity, JHEP 07 (2006) 034; arXiv:hep-th/0607138. 
[40] P. S. Landweber (Ed.), Elliptic Curves and Modular Forms in Algebraic Topology, Lect. Notes Math. 1326 (Springer-Verlag, 1988).

[41] P. S. Landweber, D. Ravenel, and R. Stong, Periodic cohomology theories defined by elliptic curves, Contemp. Math. 181 (1995) 317-338.

[42] W. Lerche, C. Vafa and N. P. Warner, Chiral rings in $\mathcal{N}=2$ superconformal theories, Nucl. Phys. B 324 (1989) 427-474.

[43] J. Li, K. Liu and J. Zhou, Topological string partition functions as equivariant indices, Asian J. Math. 10 (2006) 81-114; arXiv:math.AG/0412089].

[44] X. Ma and J. Zhou, Elliptic genera of complete intersections in weighted projective spaces, Int. J. Math. 22 (2011) 695-712; arXiv:math.AG/0411081].

[45] J. M. Maldacena, A. Strominger and E. Witten, Black hole entropy in M-theory, JHEP 12 (1997) 002; arXiv:hep-th/9711053.

[46] H. Nakajima, Lectures on Hilbert Schemes of Points on Surfaces, Amer. Math. Soc. Univ. Lect. Series 18 (1999) 1-131.

[47] N. A. Nekrasov, Seiberg-Witten prepotential from instanton counting, Adv. Theor. Math. Phys. 7 (2004) 831-864; arXiv:hep-th/0206161.

[48] S. Ochanine, Sur les genres multiplicatifs definis par des integrales elliptiques, Topology 26 (1987) 143-151.

[49] R. Pandharipande and R. P. Thomas, Stable pairs and BPS invariants, J. Amer. Math. Soc. 23 (2010) 267-297; [arXiv:math.AG/0711.3899].

[50] P. A. Perry and F. L. Williams, Selberg zeta-function and trace formula for the BTZ black hole, Int. J. Pure Appl. Math. 9 (2003) 1-21.

[51] A. Strominger and C. Vafa, Microscopic origin of the Bekenstein-Hawking entropy, Phys. Lett. B 379 (1996) 99-104; arXiv:hep-th/9601029.

[52] C. Vafa and E. Witten, A strong coupling test of S-duality, Nucl. Phys. B 431 (1994) 3-77; arXiv:hep-th/9408074.

[53] C. Vafa, Instantons on D-branes, Nucl. Phys. B 463 (1996) 435-442; arXiv:hep-th/9512078.

[54] N. P. Warner, $\mathcal{N}=2$ supersymmetric integrable models and topological field theories, ICTP Ser. Theor. Phys. 9 (1993) 143-179; arXiv:hep-th/9301088.

[55] E. Witten, Elliptic genera and quantum field theory, Commun. Math. Phys. 109 (1987) $525-536$.

[56] E. Witten, On Landau-Ginzburg description of $\mathcal{N}=2$ minimal models, Int. J. Mod. Phys. A 9 (1994) 4783-4800. 